\title{
Stellar evolution and nucleosynthesis
}

\section{- Low- and intermediate mass stars -}
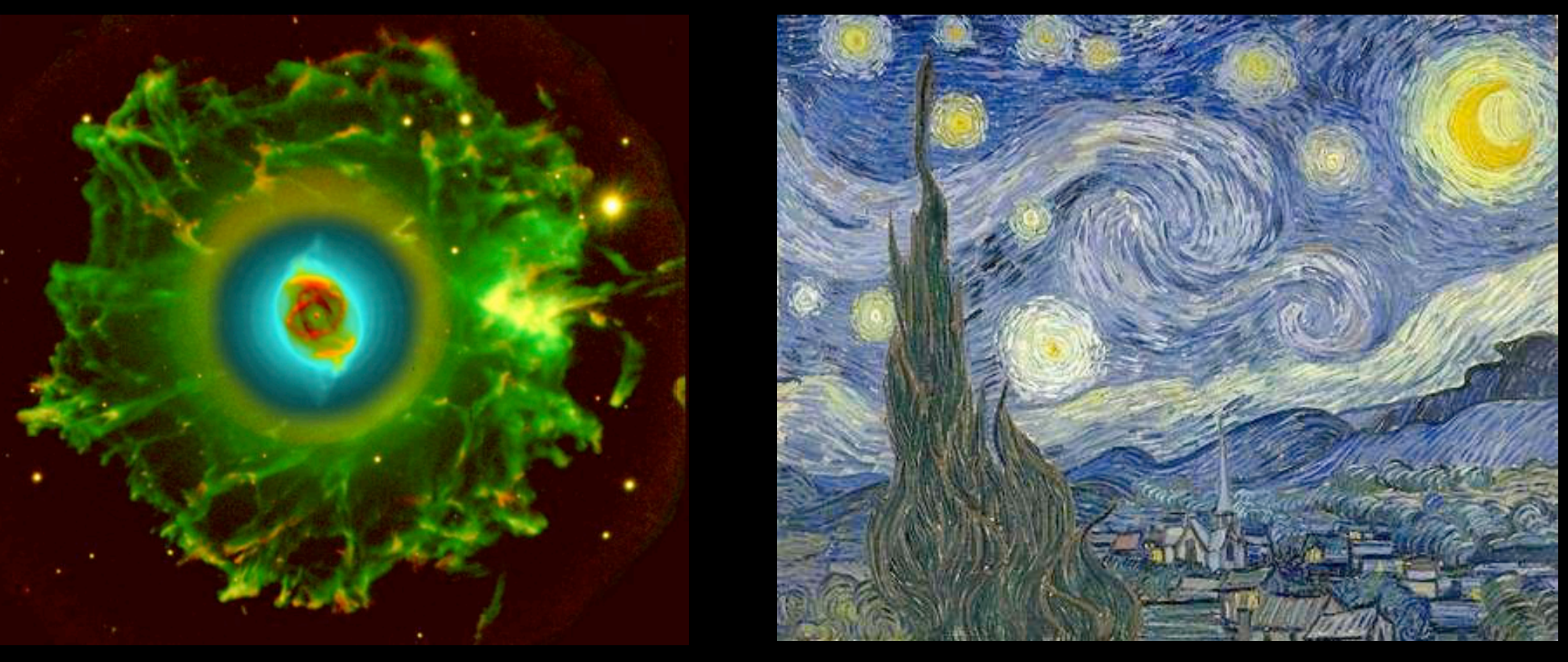

Corinne Charbonnel

CNRS \& Geneva Observatory 


\section{Stellar evolution and nucleosynthesis}

\section{- Low and intermediate-mass stars -}

- Evolution and nucleosynthesis in stars :

A global overview of the hydrostatic phases

Diagrams : HRD, $\log$ Tc vs $\log \rho c$, main evolution and nucleosynthetic phases, mass limits for the various nucleosynthetic paths

- Main sequence nucleosynthesis - Clues from ${ }^{3} \mathrm{He}$

- Nucleosynthesis in AGB stars

AGB structure, TP, mass loss, HBB, 3d dredge-up, rotation, processus-s, yields

Constraints from $\mathrm{PNe}$ and post-AGBs 
From $\sim 0.9$ to $8 \mathrm{M}_{\odot}$

\section{Thermąlly Pulsing AGB}

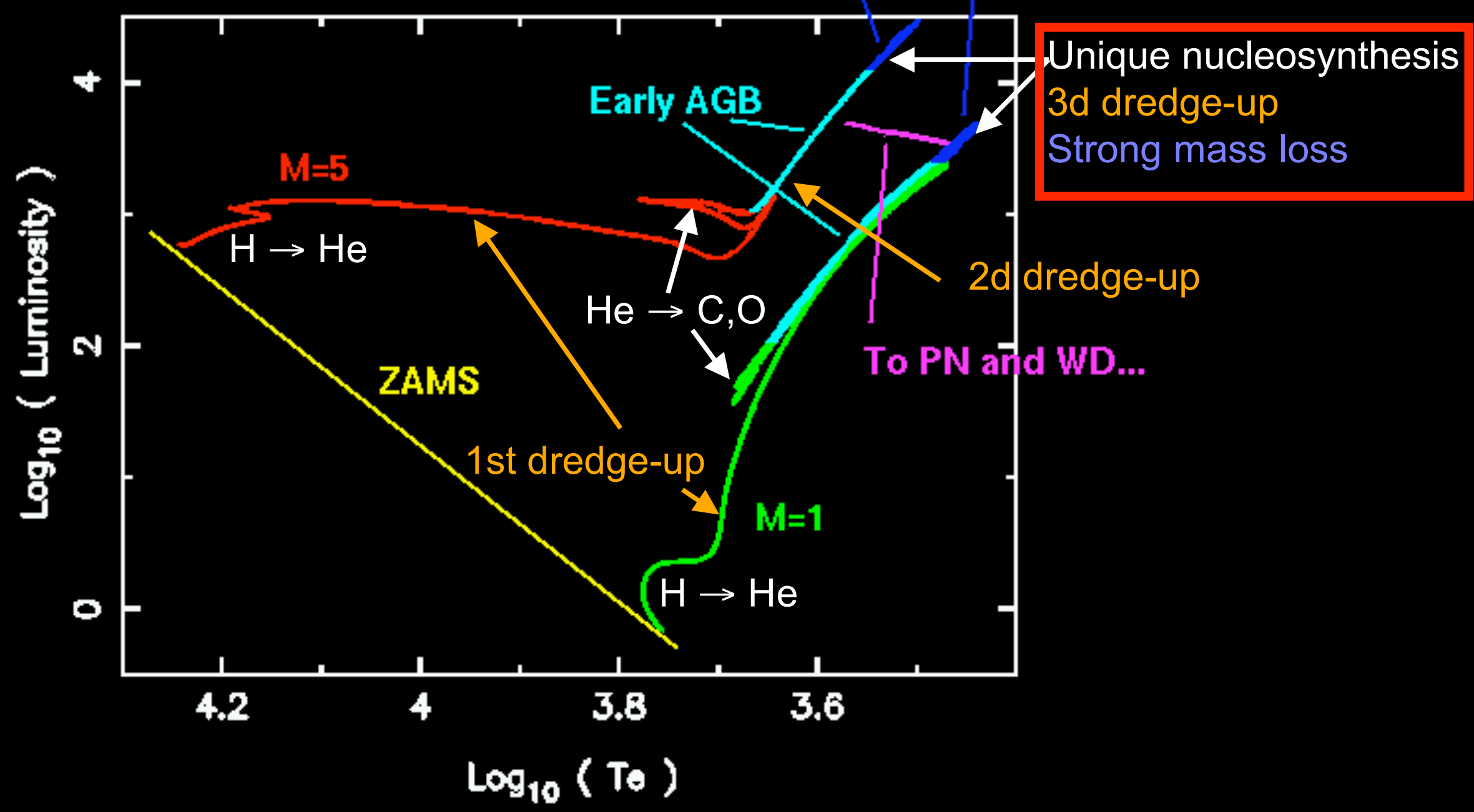

Adapted from Lattanzio 

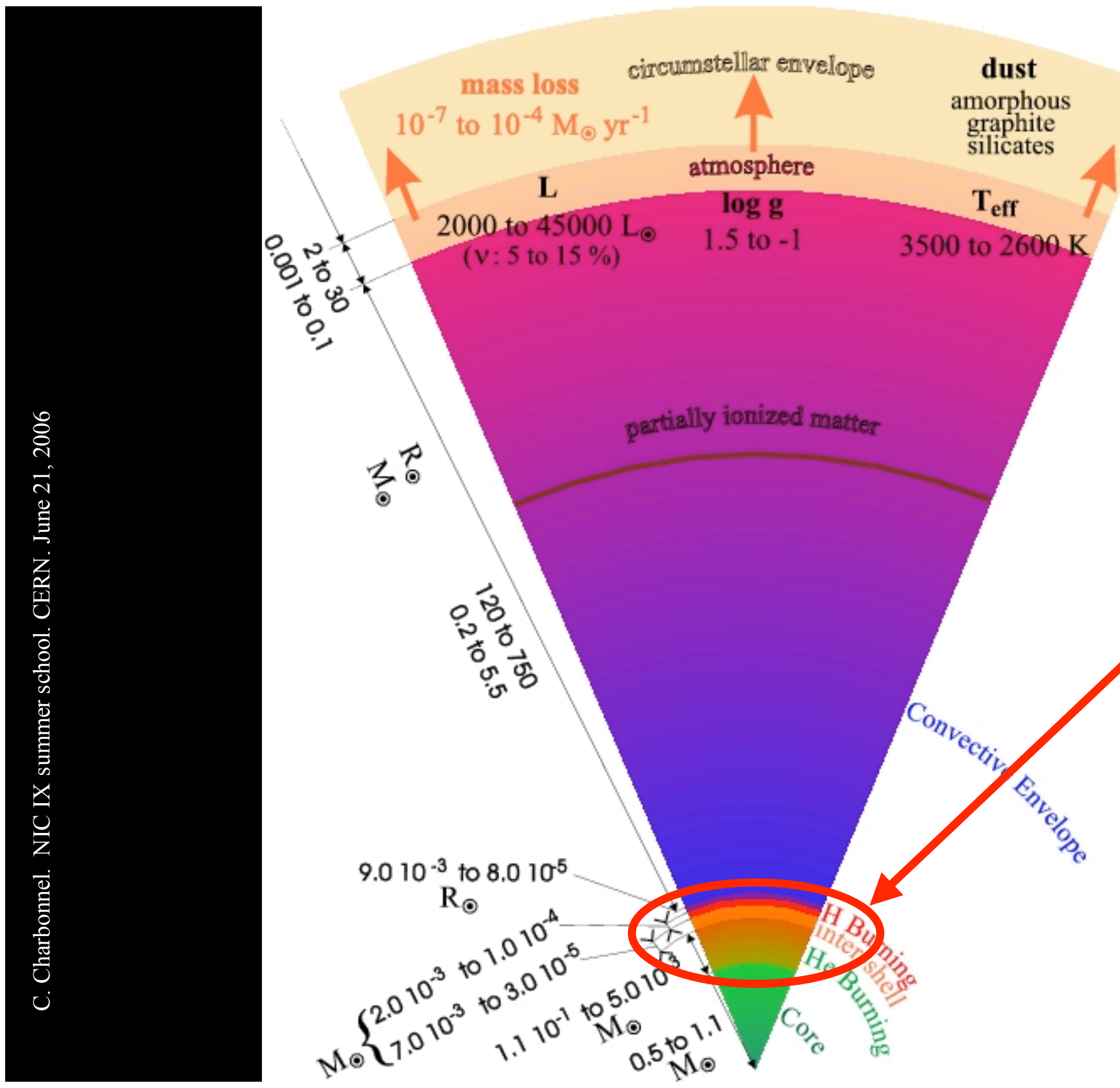

Figure by M.Forestini 


\section{Global Structure of an AGB star}

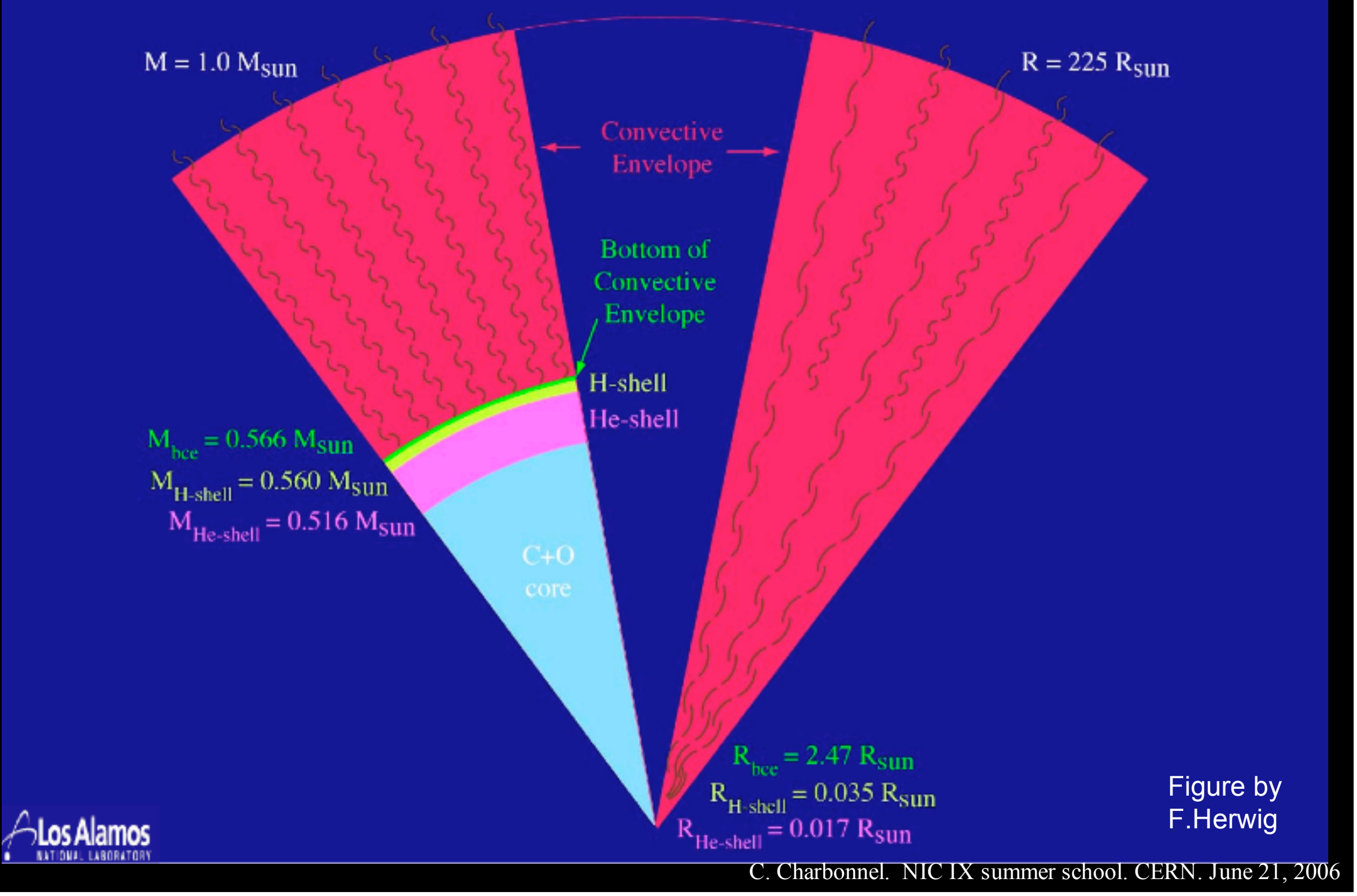




\section{Kippenhahn diagram on TP-AGB phase}

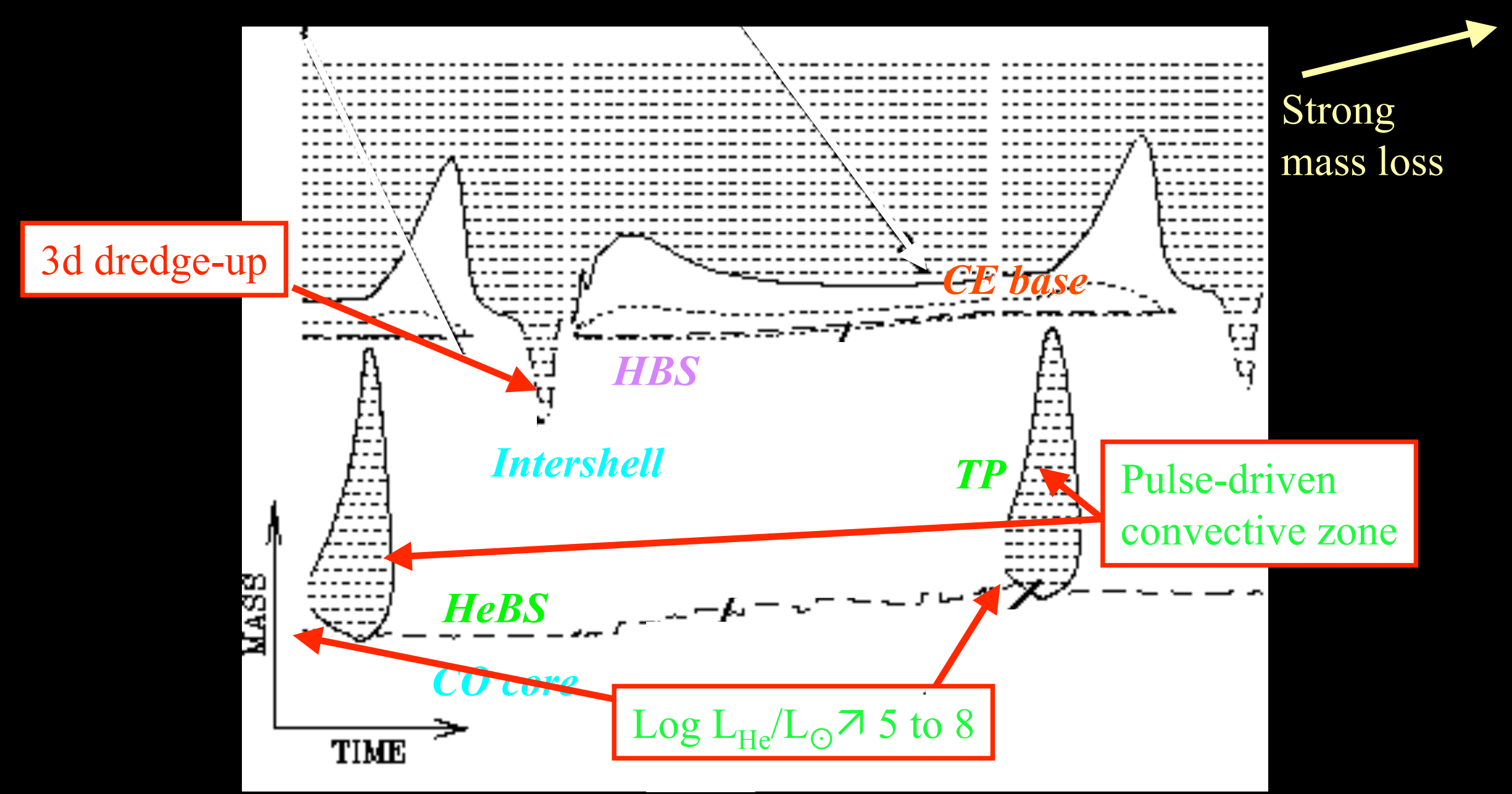

Adapted from

N.Mowlavi 


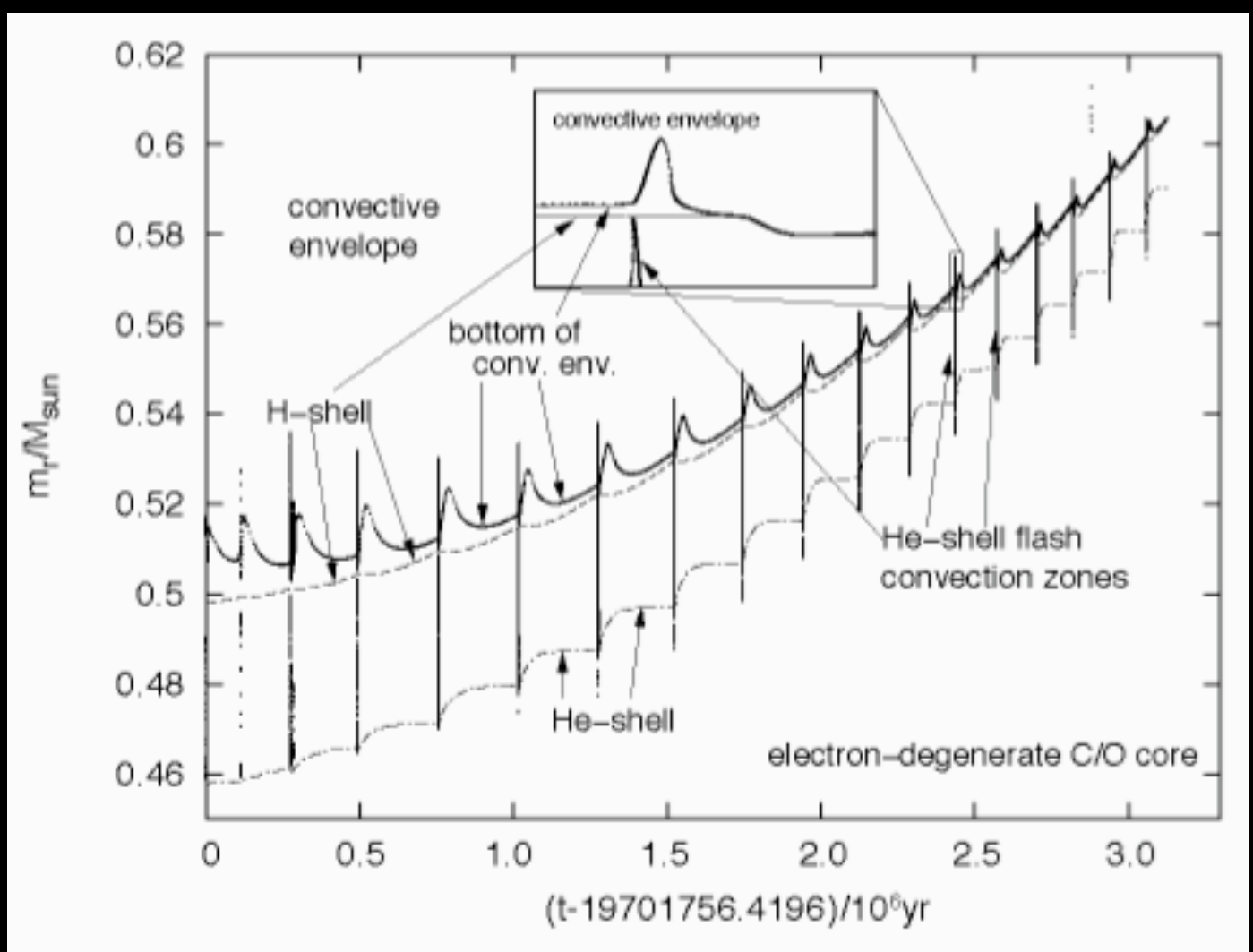

Figure by F.Herwig 


\section{Nucleosynthesis on the TP-AGB}


Transport of H into C-rich layers

Radiative s-process via ${ }^{13} \mathrm{C}(\alpha, \mathrm{n}){ }^{16} \mathrm{O}$ during the interpulse

Ashes of the interpulse HBS engulfed into the pulse convective tongue

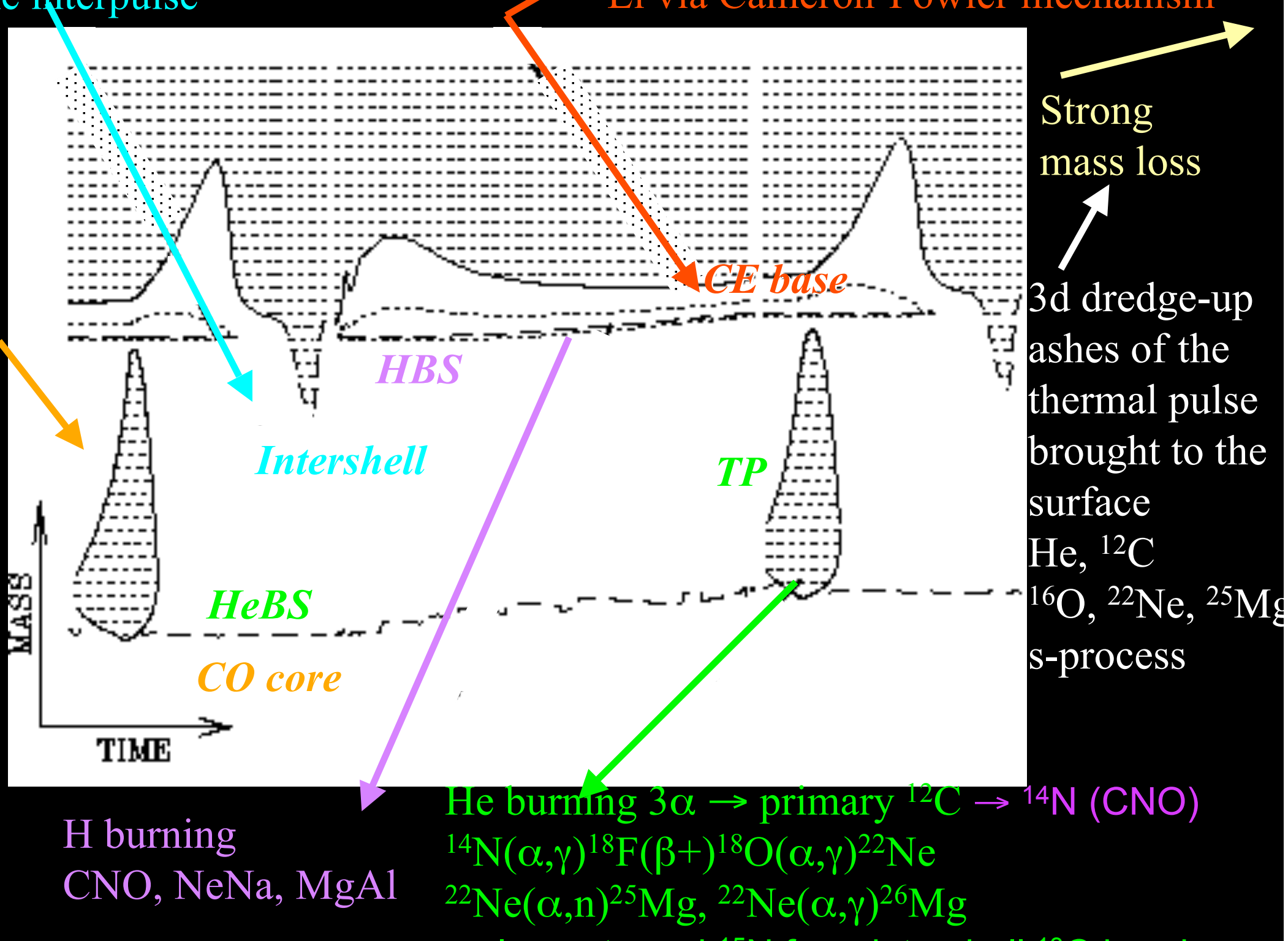

s-elements and ${ }^{15} \mathrm{~N}$ from intershell ${ }^{13} \mathrm{C}$ burning 
$\checkmark$ Third dredge-up $\left(\mathrm{M} \geq 1.5 \mathrm{M}_{\odot}\right.$ at $\left.\mathrm{Z}_{\odot}\right)$ products of He-burning in the TP ${ }^{4} \mathrm{He},{ }^{12} \mathrm{C},{ }^{16} \mathrm{O},{ }^{22} \mathrm{Ne},{ }^{25} \mathrm{Mg}$, s-process elements increase

$\checkmark$ Hot-bottom burning $\left(\mathrm{M} \geq 4-4.5 \mathrm{M}_{\odot}\right)$

$\mathrm{CN}$-cycle $:{ }^{12} \mathrm{C} \rightarrow{ }^{13} \mathrm{C} \rightarrow{ }^{14} \mathrm{~N}$

ON-cycle $:{ }^{16} \mathrm{O} \rightarrow{ }^{14} \mathrm{~N}$

Predictions depend on

$\checkmark$ Stellar parameters

$$
\mathrm{M}, \mathrm{Z}
$$

$\checkmark$ Input physics prescriptions

Nuclear reaction rates, opacities, ...

$\checkmark$ Various incompletely understood physical parameters

Mass loss, convection, transport processes, rotation, ... which rests on semiempirical calibrations

(e.g. C star luminosity function, initial-final M relation) that have to be extrapolated

to a range of $\mathrm{M}, \mathrm{Z}$ for which no empirical data are available 


\section{Illustration}

of the uncertainties

on the yields

$\rightarrow$ Mass loss 
Mass loss affects the AGB duration, i.e.

the number and strength of TPs and subsequent $3 \mathrm{~d}$ DUP events and the growth of Mcore

A minimum $\mathrm{M}_{\text {envelop }}$ is required for HBB and 3d DUP to occur HBB may be shut down long before $3 d$ DUP ends

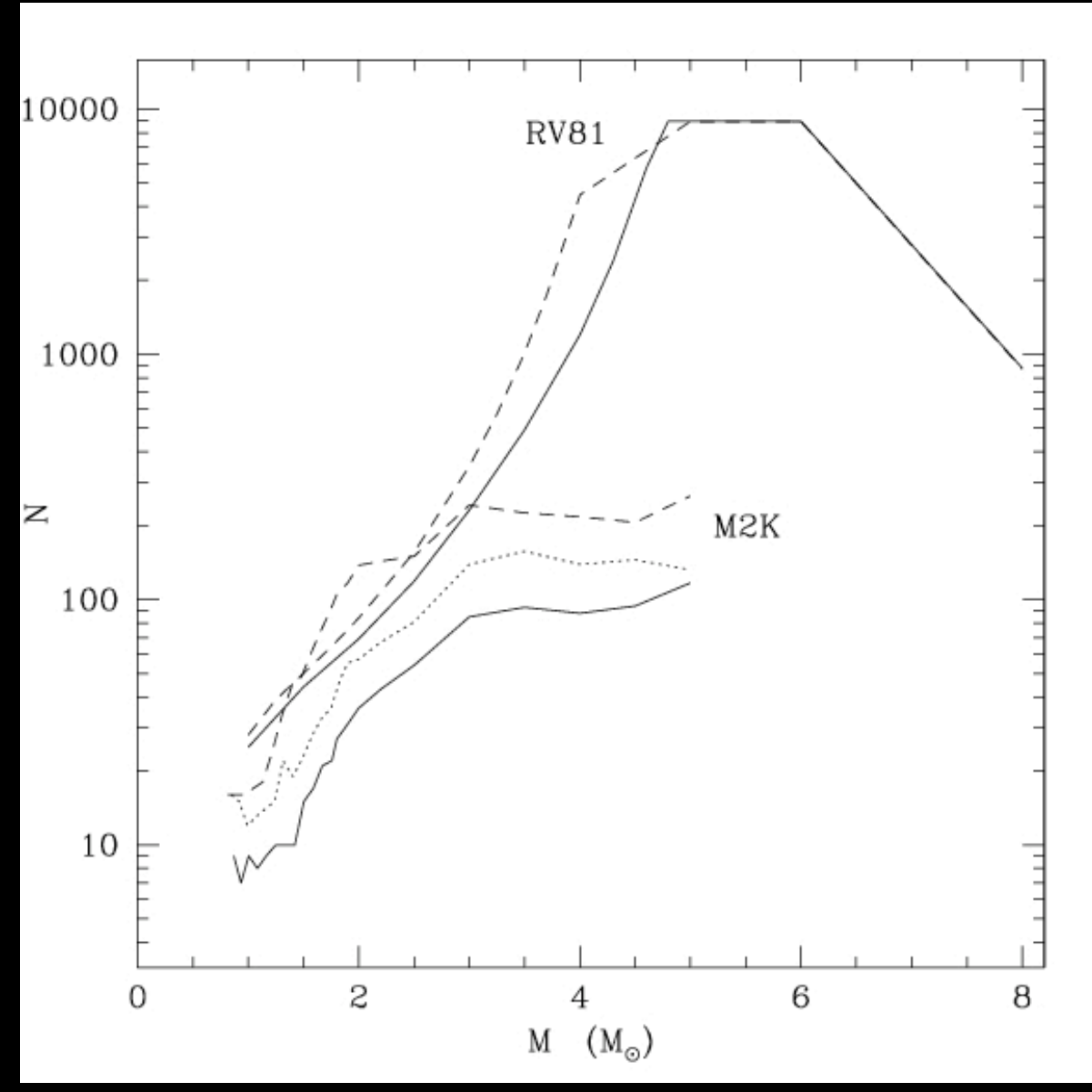

Expected number of TP vs initial $\mathbf{M}^{*}$

Renzini \& Voli (81)

Reimers law (75), no superwind

Marigo (00)

Vassiliadis \& Wood (93)

$\begin{aligned} \mathrm{Z}=\mathrm{Z}_{\odot} & \\ 0.008 & \ldots \ldots \ldots \\ 0.004 & \boldsymbol{-}\end{aligned}$

Marigo (00) 
Mass loss affects the AGB duration, i.e.

the number and strength of TPs and subsequent $3 \mathrm{~d}$ DUP events and the growth of Mcore

A minimum $\mathrm{M}_{\text {envelop }}$ is required for HBB and 3d DUP to occur HBB may be shut down long before $3 d$ DUP ends

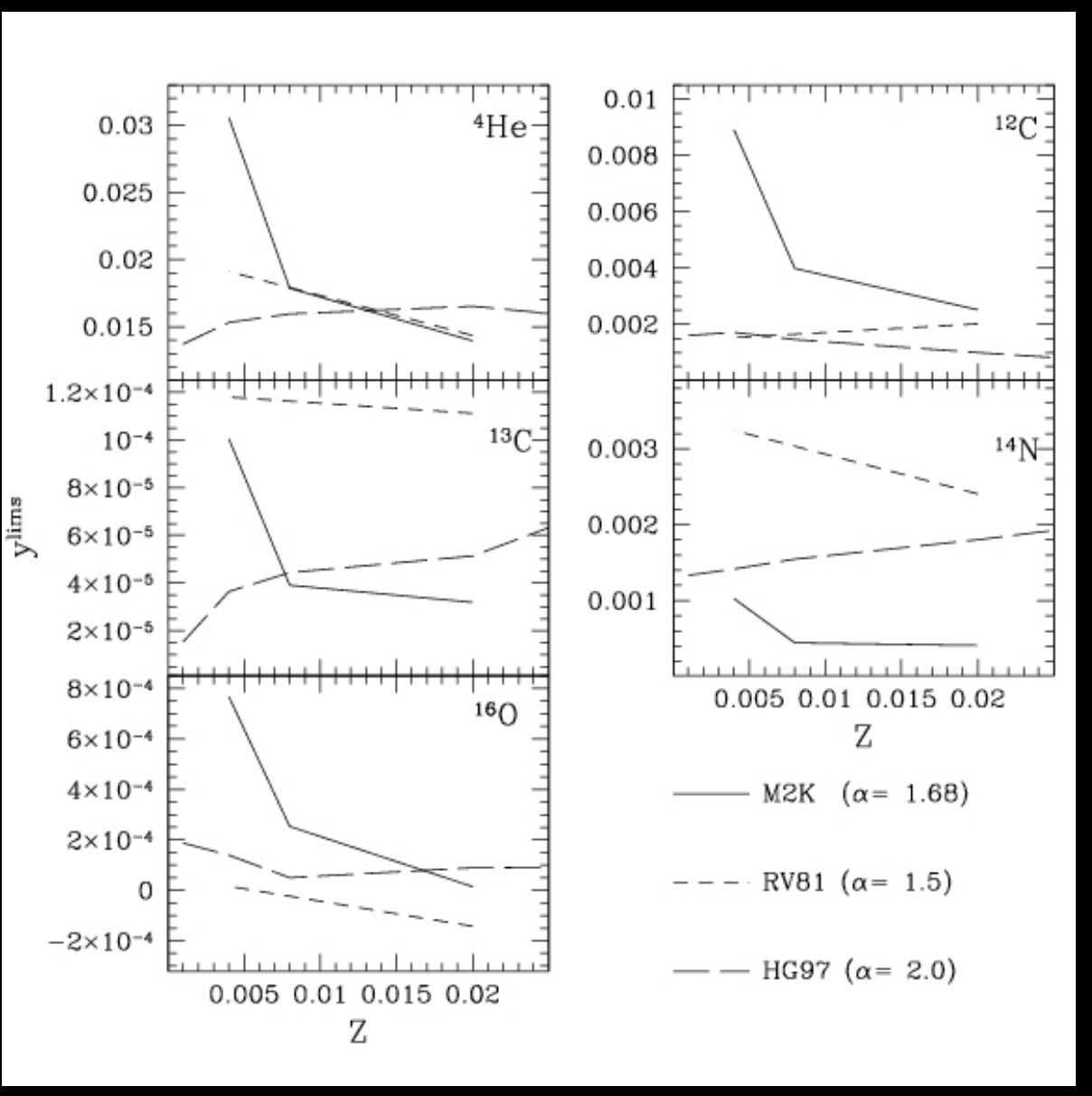

Integrated yields as a function of $\mathbf{Z}$

Renzini \& Voli (81) - - -

Reimers law (75), no superwind

Marigo (00)

Vassiliadis \& Wood (93) 


\section{Illustration}

\section{of the uncertainties on the yields}

$\rightarrow$ The case of $\mathrm{O}$ and $\mathrm{Na}$ 


\section{HBB ( $\mathrm{M}>4$ Msun)}

\section{CNO, NeNa}

At higher $\mathrm{T}, \underline{\underline{23}} \underline{\mathrm{Na}}$ destroyed again via ${ }^{23} \mathrm{Na}(\mathrm{p}, \gamma)^{24} \mathrm{Mg}$

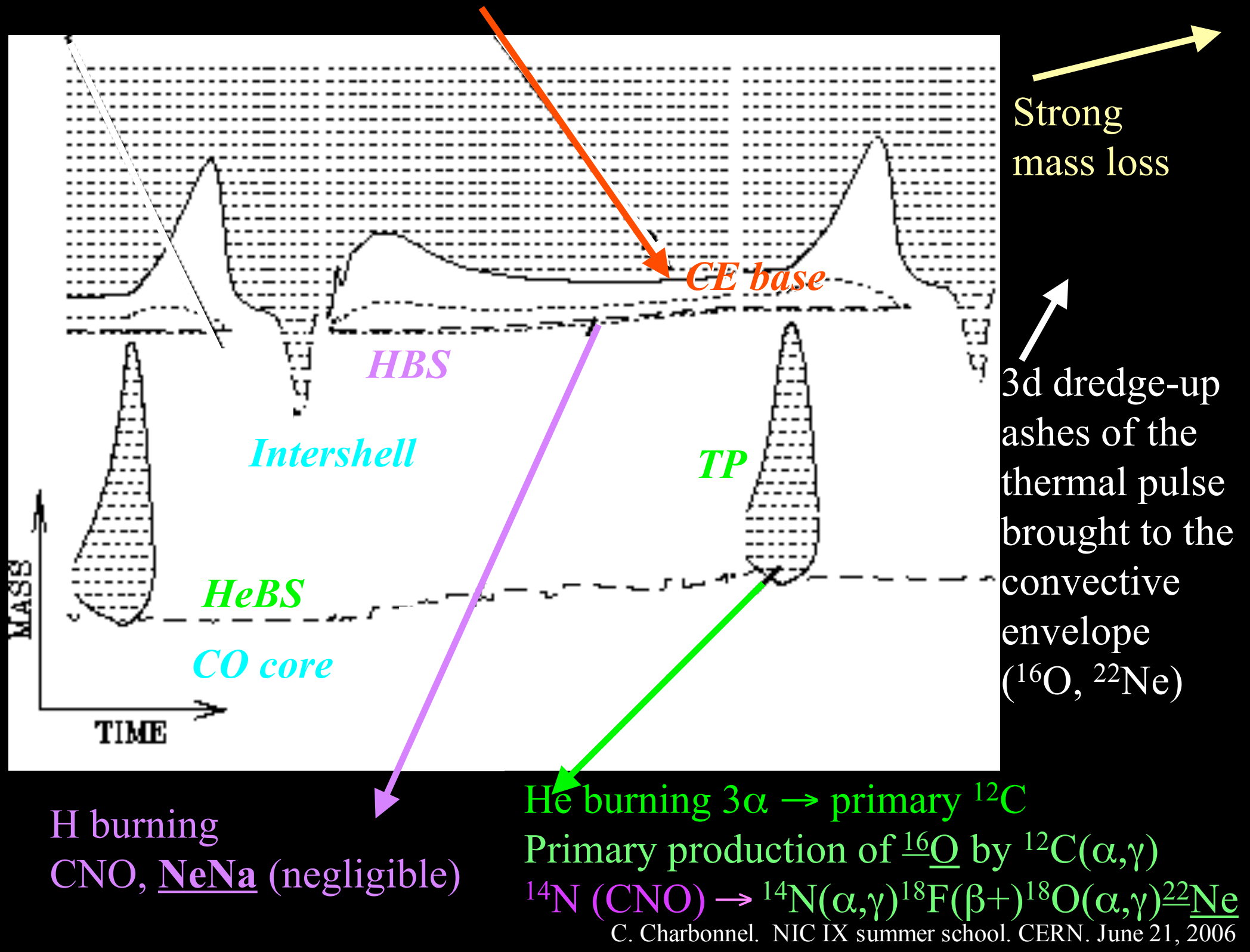




\section{Illustration}

of the uncertainties on the yields

$\rightarrow$ HBB and 3d DUP 


\section{O, Na evolution at the surface of a low-Z massive TP-AGB star}

Delicate interplay of $3 \mathrm{~d}$ dredge-up and hot bottom burning

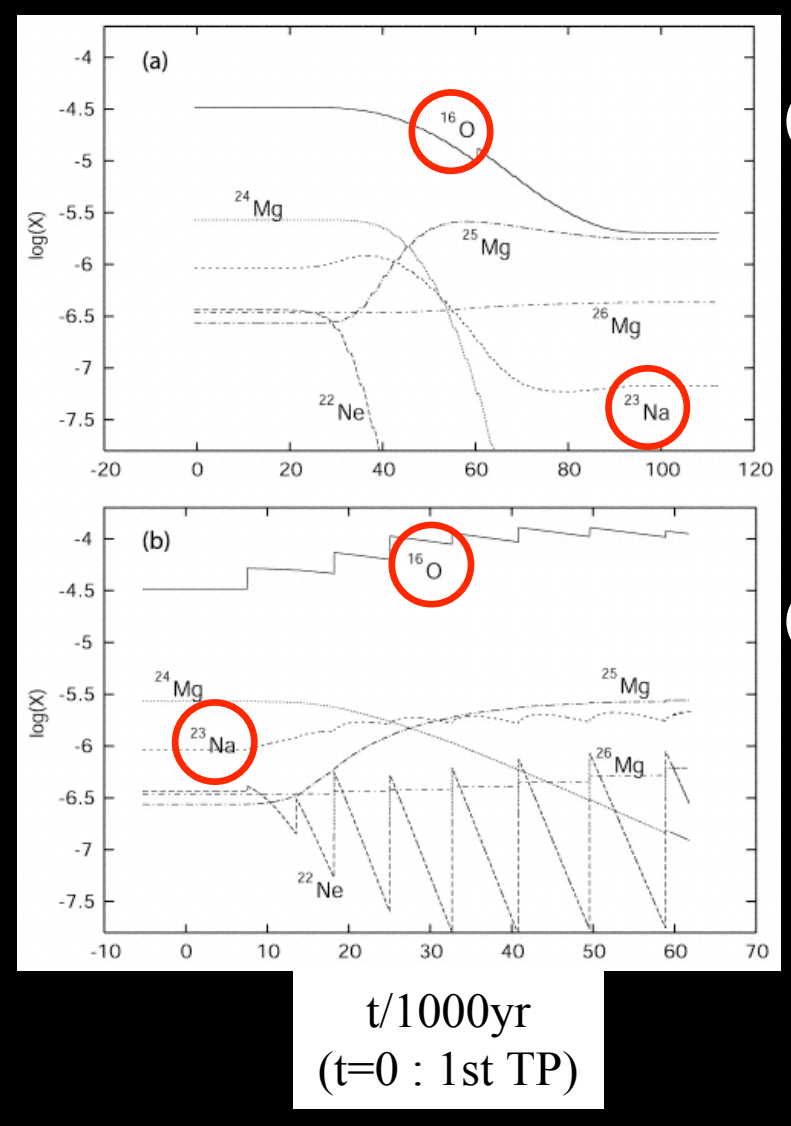

(a) No 3DUP, only HBB

$\rightarrow$ Large ${ }^{16} \mathrm{O}$ depletion

$\rightarrow{ }^{23} \mathrm{Na}$ depletion

(due to the lack of ${ }^{22} \mathrm{Ne}$ dredged-up)

(b) Strong 3DUP, HBB, no mass loss

$\rightarrow 3$ DUP of the ${ }^{16} \mathrm{O}$-rich layers below the TP

$\rightarrow{ }^{23} \mathrm{Na}$ increase (from dredged-up ${ }^{22} \mathrm{Ne}$ )

Denissenkov \& Herwig (03) 


\section{Illustration}

\section{of the uncertainties on the yields}

$\rightarrow$ Convection 


\section{Impact of convection}

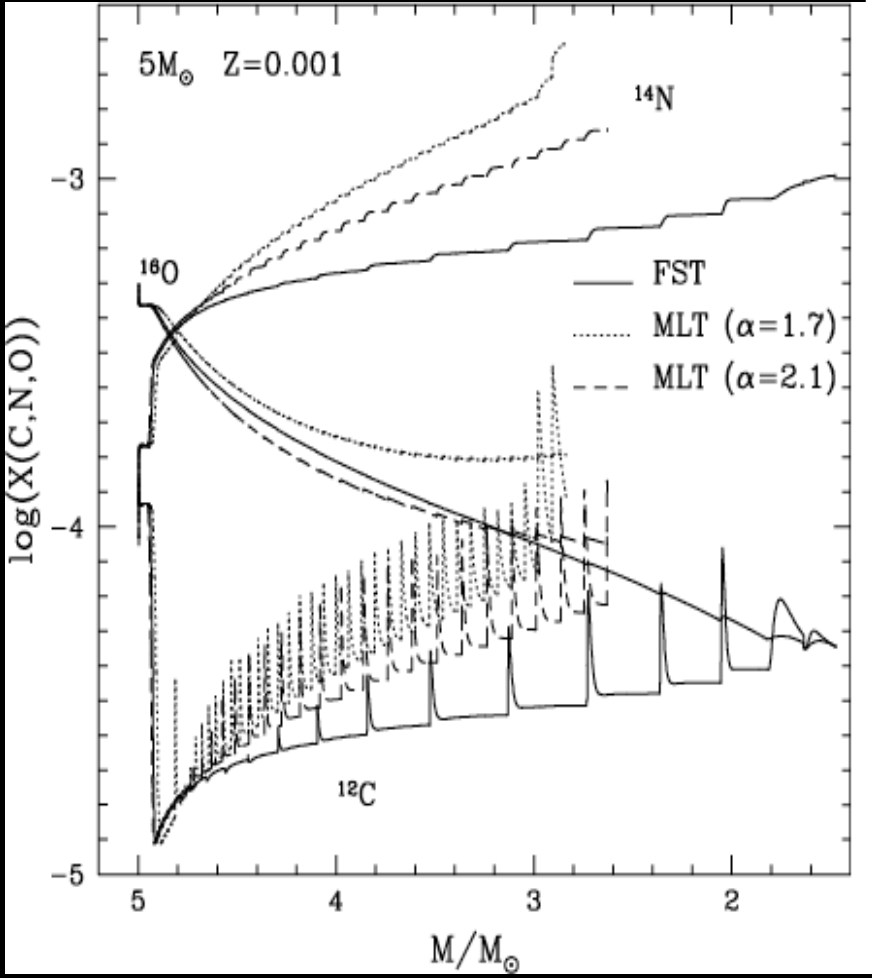

Ventura \& D'Antona (05 I)

See also Renzini \& Voli (81), Sackmann \& Boothroyd (91), Blöcker \& Schönberner (91), D’Antona \& Mazzitelli (96)

Full Spectrum of Turbulence (Canuto \& Mazzitelli 91) $\rightarrow$ much more efficient HBB than with MLT (on the AGB : higher L, stronger mass loss)

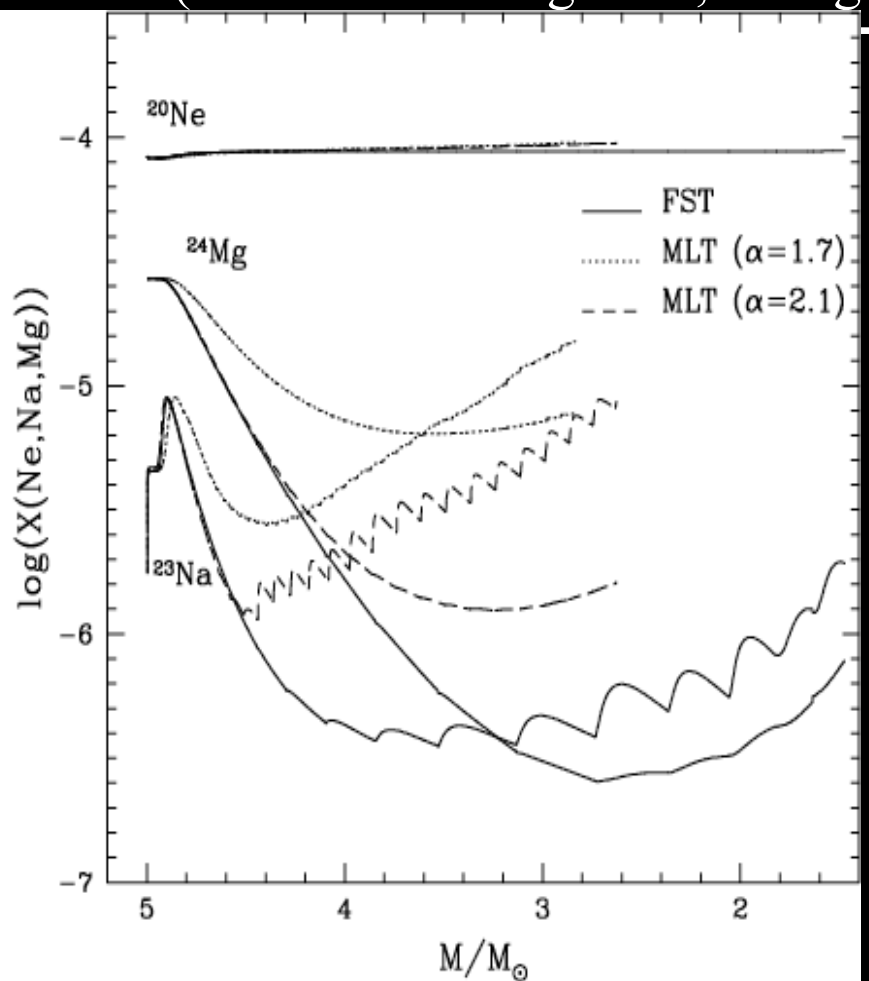

\section{MLT17:}

Little O depletion (factor of $\sim 2$ ) Extremely large increase of $\mathrm{Na}$ and $\mathrm{N}$ $\mathrm{C}+\mathrm{N}+\mathrm{O}$ increase by $\sim 0.8 \mathrm{dex}$

FST :

Largest $\mathrm{O}$ depletion Slight decrease of $\mathrm{Na}$ $\mathrm{C}+\mathrm{N}+0$ conserved 


\section{Impact}

\section{of convection \\ on nucleosynthesis}

Ventura \& D’Antona (05 II)

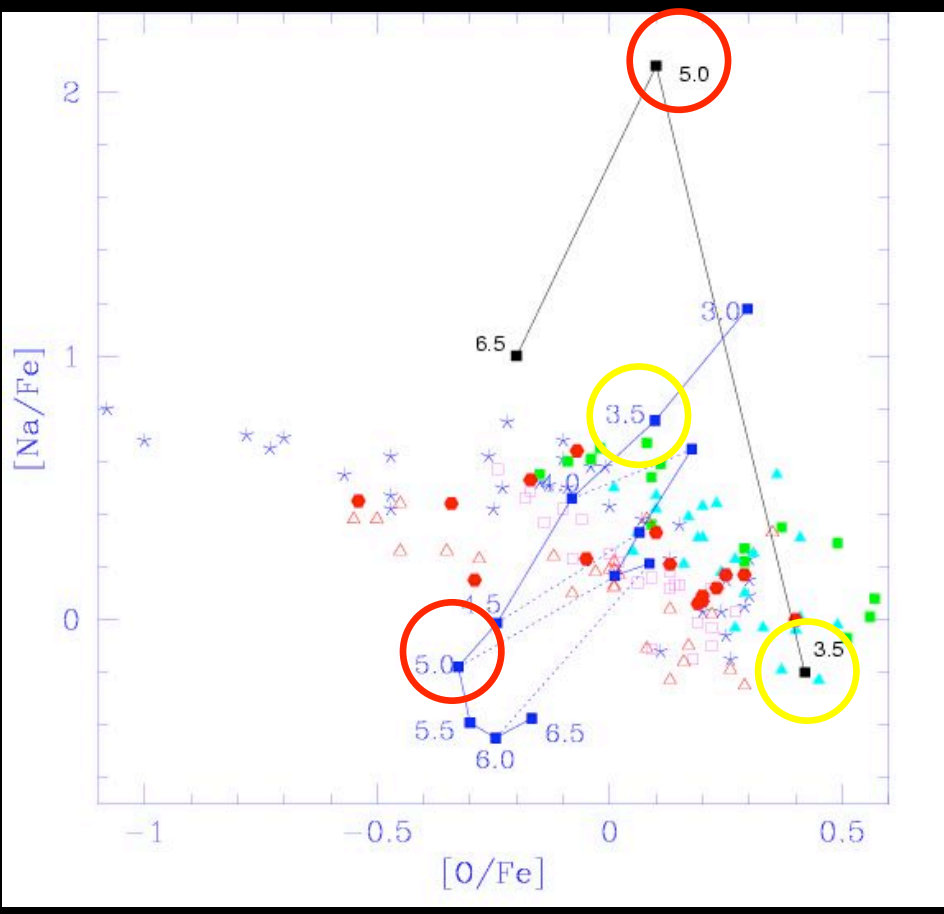

Fenner et al.(04)

Overproduction of (primary) ${ }^{23} \mathrm{Na}$

due to the burning of dredged-up ${ }^{20} \mathrm{Ne}$

Ventura \& D'Antona (05II)

Underproduction of ${ }^{23} \mathrm{Na}$

due to smaller number of 3DUP episodes and larger $\mathrm{T}$

Both sets are unable to reproduce the data

« The predictive power of AGB models is still undermined by many uncertainties 》 (VD'A05) 


\section{Illustration}

of the uncertainties

on the yields

$\rightarrow$ Rotation

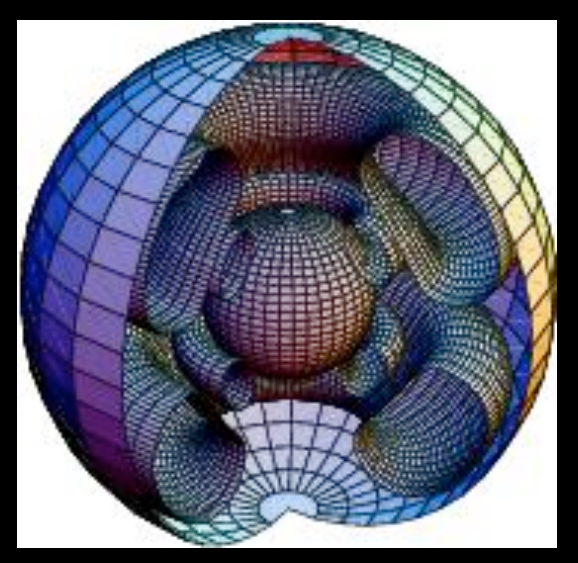




\section{Rotating AGB models}

Decressin, Charbonnel, Siess, Palacios, Meynet (06)

\section{STAREVOL}

Meridional circulation and shear turbulence

Zahn (92), Chaboyer \& Zahn (95)

Talon \& Zahn (97), Maeder \& Zahn (98)

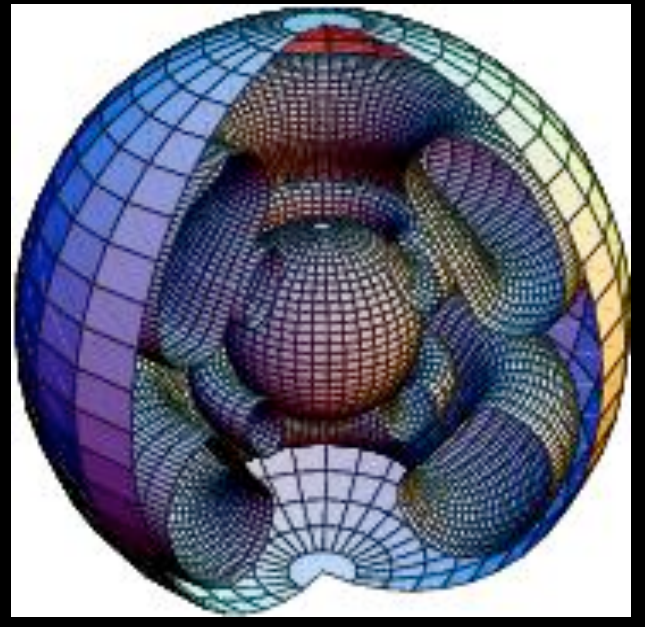

Courtesy of

Georges Meynet

Same physics successfully applied to

Massive stars : HeBCN anomalies (see references in Maeder \& Meynet 00) Low-mass stars : Hot side of the Li dip, Li in subgiants (Charbonnel \& Talon 99, 


\section{Rotating AGB models}

Decressin, Charbonnel, Siess, Palacios, Meynet (06)

$7 \mathrm{M}_{\odot}$ star, $\mathrm{Z}=10^{-5}$

Profiles at the end of central He-burning
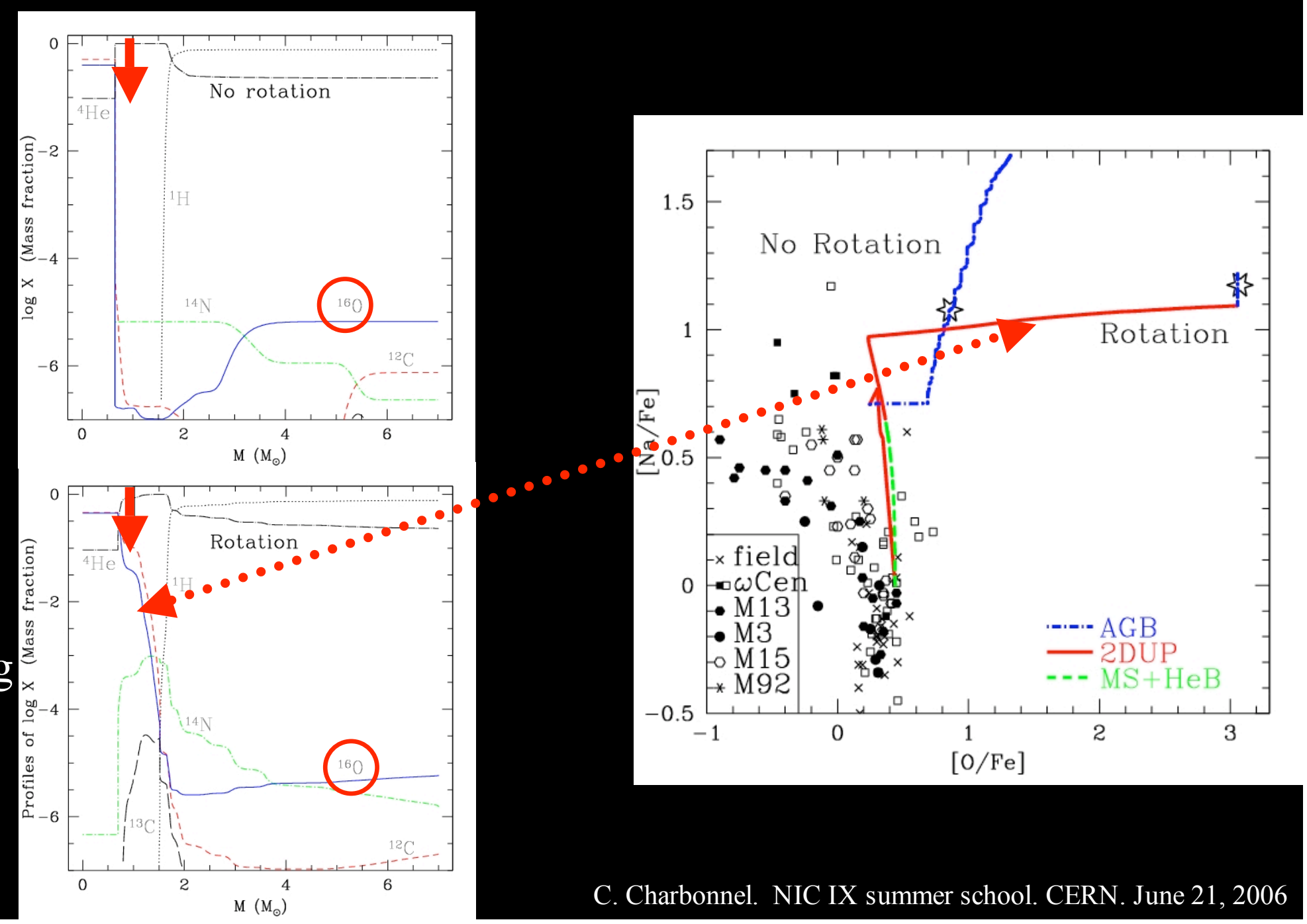


\section{Primary ${ }^{14} \mathrm{~N}$}

in rotating intermediate-mass stars
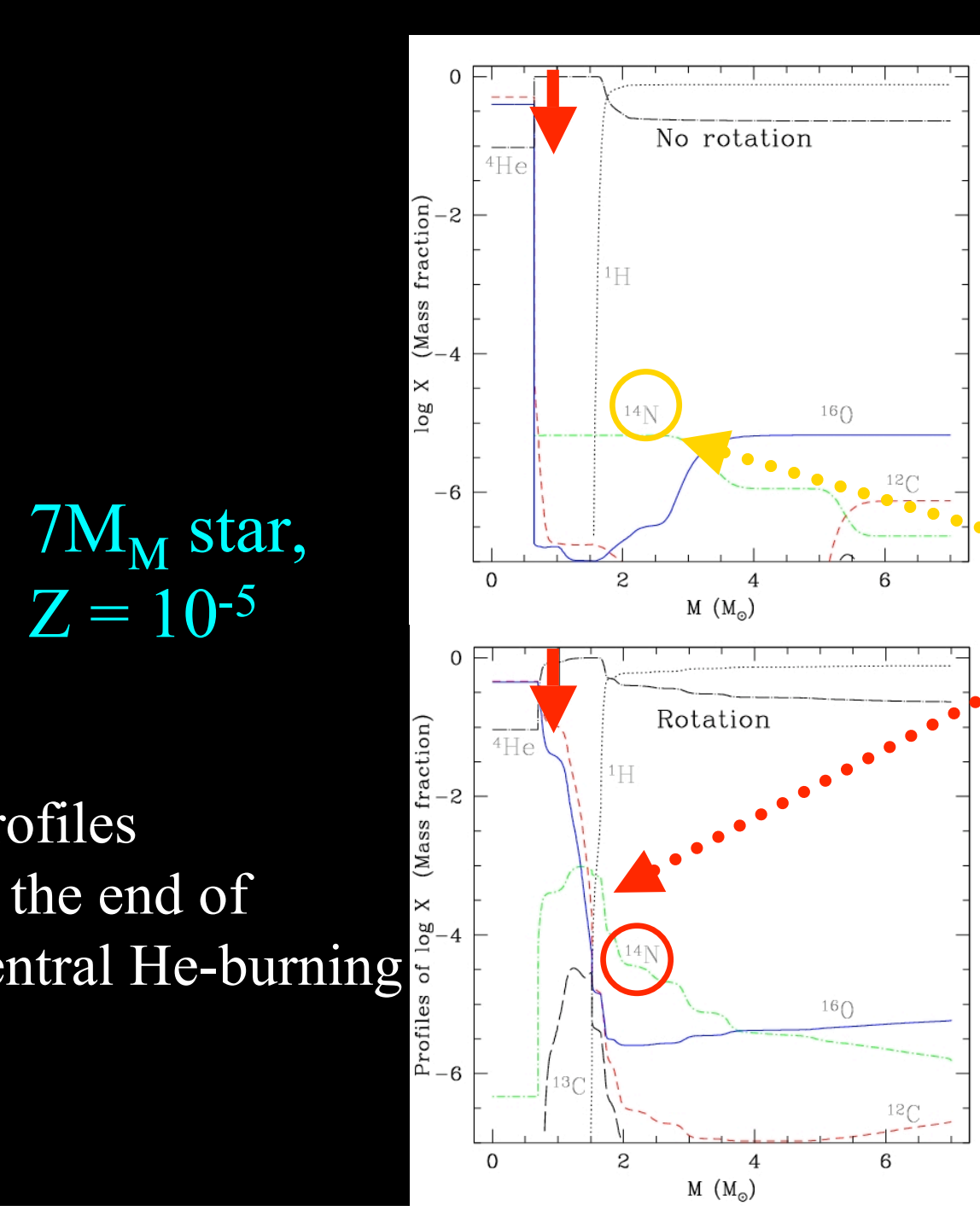

Meynet \& Maeder (02)

Profiles

at the end of

central He-burning

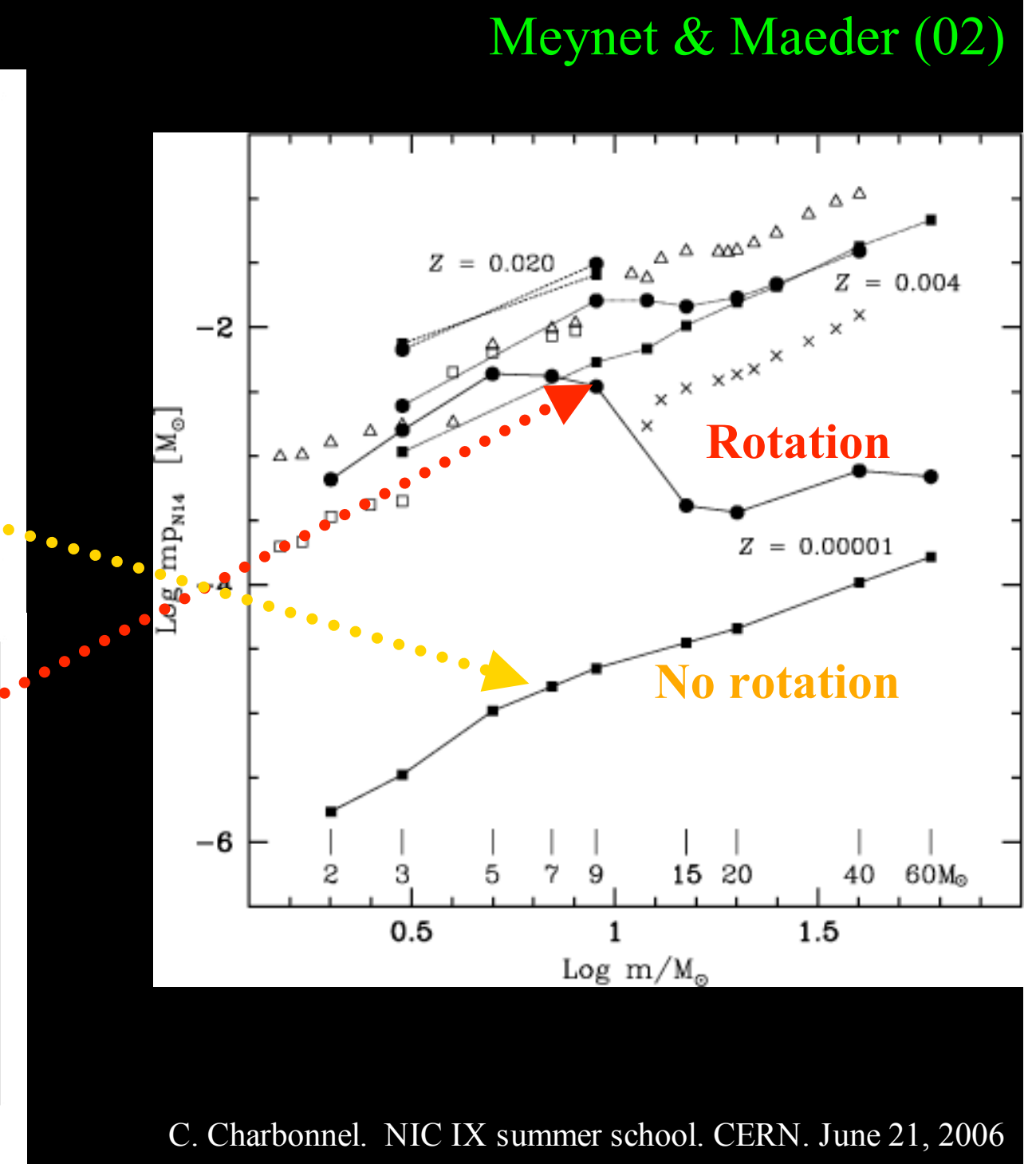




\title{
The importance of PNe
}

\author{
as constraints
}

\section{on nucleosynthesis}




\section{Insight on the nucleosynthetic properties of the PNe progenitors}

$\checkmark$ C, N : Efficiency of 3d DUP vs HBB

$\checkmark$ He : Cumulative effect of the 1st, $2 \mathrm{~d}$, $3 \mathrm{~d}$ dredge-up, and HBB

$\checkmark \mathrm{O}:$ Composition of the TP, efficiency of HBB

$\checkmark \mathrm{Ne}:$ Synthesis during the TP, efficiency of HBB, s-process ${ }^{22} \mathrm{Ne}(\alpha, n){ }^{25} \mathrm{Mg}$

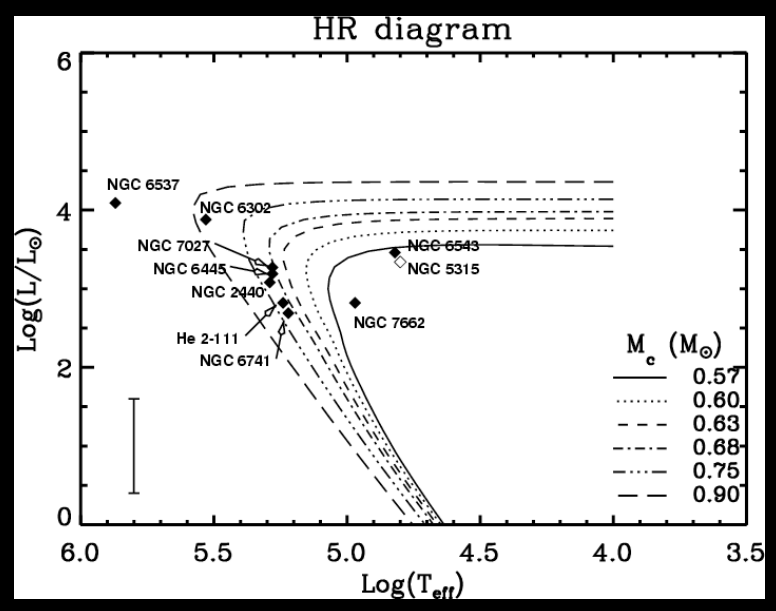

Marigo et al. (03) vs Grevesse \& Sauval (98) $+O$ from Allende-Prieto et al. (01)

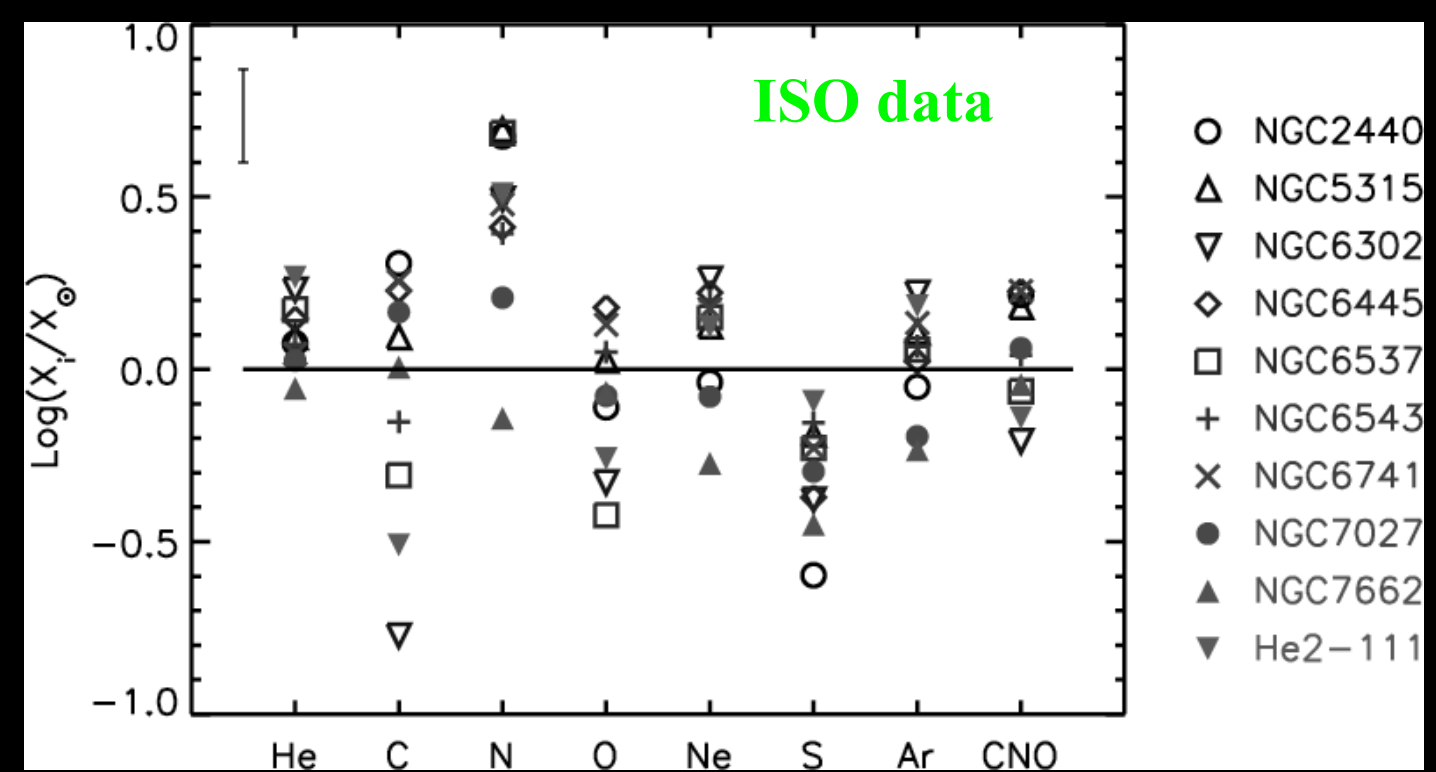




\section{Insight on the nucleosynthetic properties of the PNe progenitors}

$\checkmark \mathrm{C}, \mathrm{N}:$ Efficiency of $3 \mathrm{~d}$ DUP vs HBB

$\checkmark$ He : Cumulative effect of the 1st, $2 \mathrm{~d}$, $3 \mathrm{~d}$ dredge-up, and HBB

$\checkmark \mathrm{O}:$ Composition of the TP, efficiency of HBB

$\checkmark \mathrm{Ne}:$ Synthesis during the TP, efficiency of HBB, s-process ${ }^{22} \mathrm{Ne}(\alpha, n){ }^{25} \mathrm{Mg}$

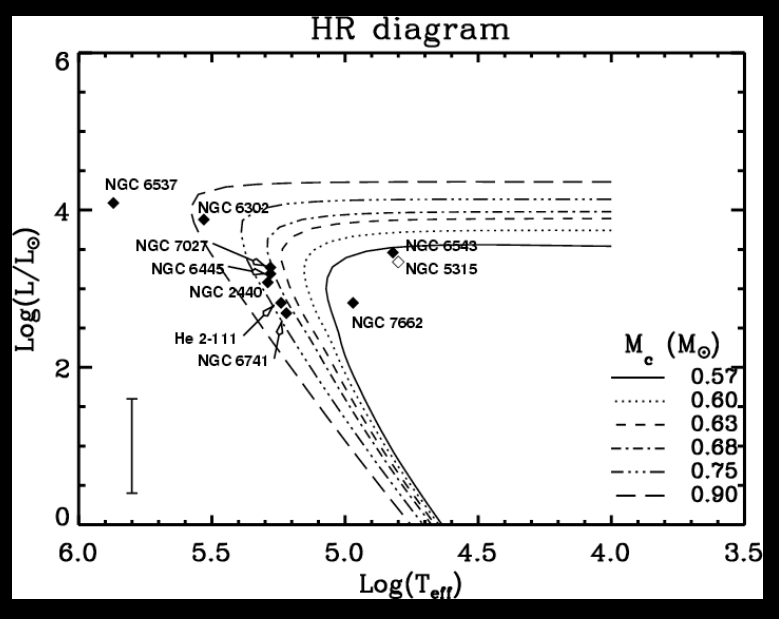

Marigo et al. (03) vs Asplund et al. (05)

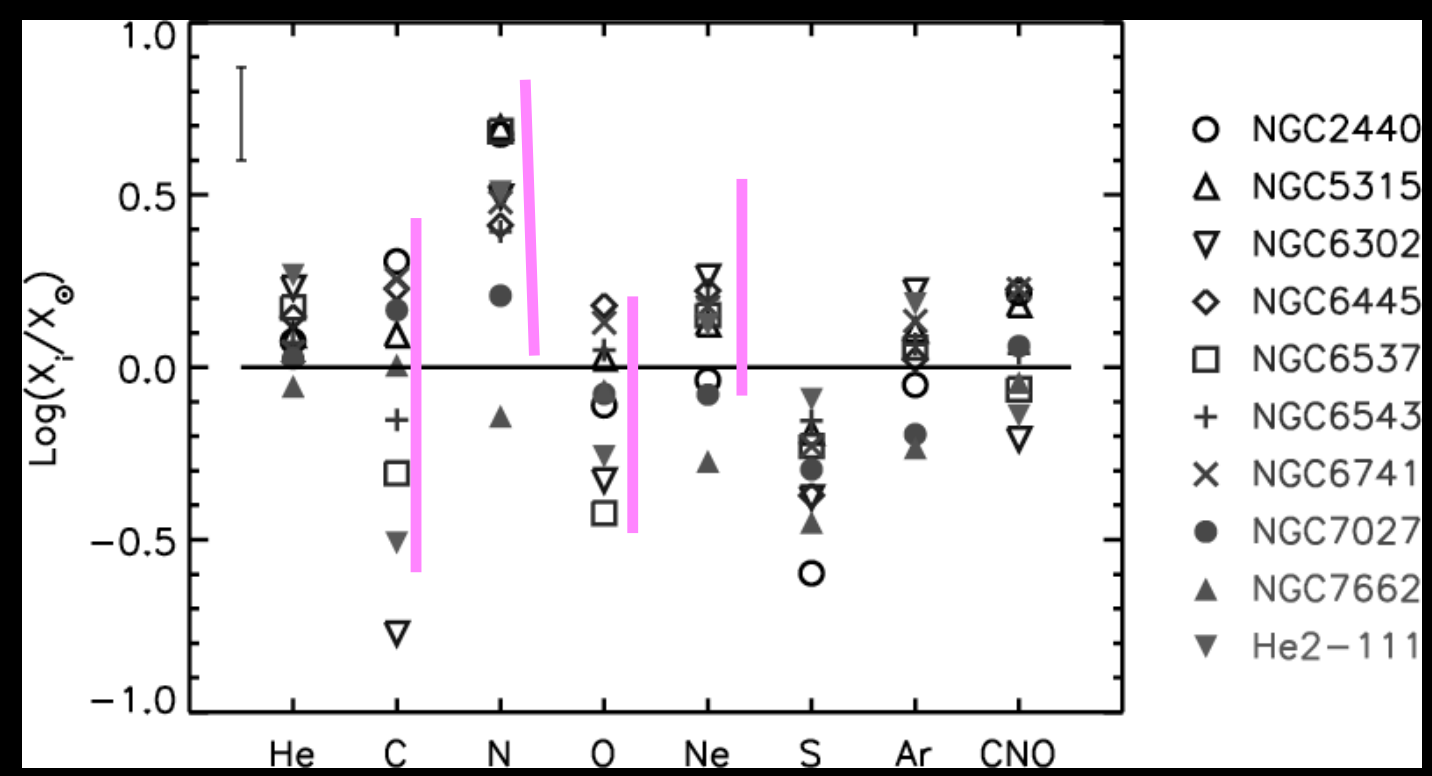

C. Charbonnel. NIC IX summer school. CERN. June 21, 2006 


\section{The s-process}




\section{s-process}

$(\mathrm{Z}, \mathrm{A})+\mathrm{n} \rightarrow(\mathrm{Z}, \mathrm{A}+1)+\gamma$

If $(Z, A+1)$ is stable, it will capture another neutron latter on If $(Z, A+1)$ is unstable :

With a low neutron flux, $(\mathrm{Z}, \mathrm{A}+1)$ may decay before next n-capture $(Z, A+1) \rightarrow \beta^{-}+v+(Z+1, A+1)$ : s-process

Nuclei symetric in proton and neutron numbers With a strong neutron flux,

$$
(\mathrm{Z}, \mathrm{A}+1)+\mathrm{n} \rightarrow(\mathrm{Z}, \mathrm{A}+2)+\gamma \quad: \text { r-process }
$$

In the s-process the n-captures are slower than subsequent $\beta$-decays.

Typical neutron densities are $7<\log N_{n}<10$.

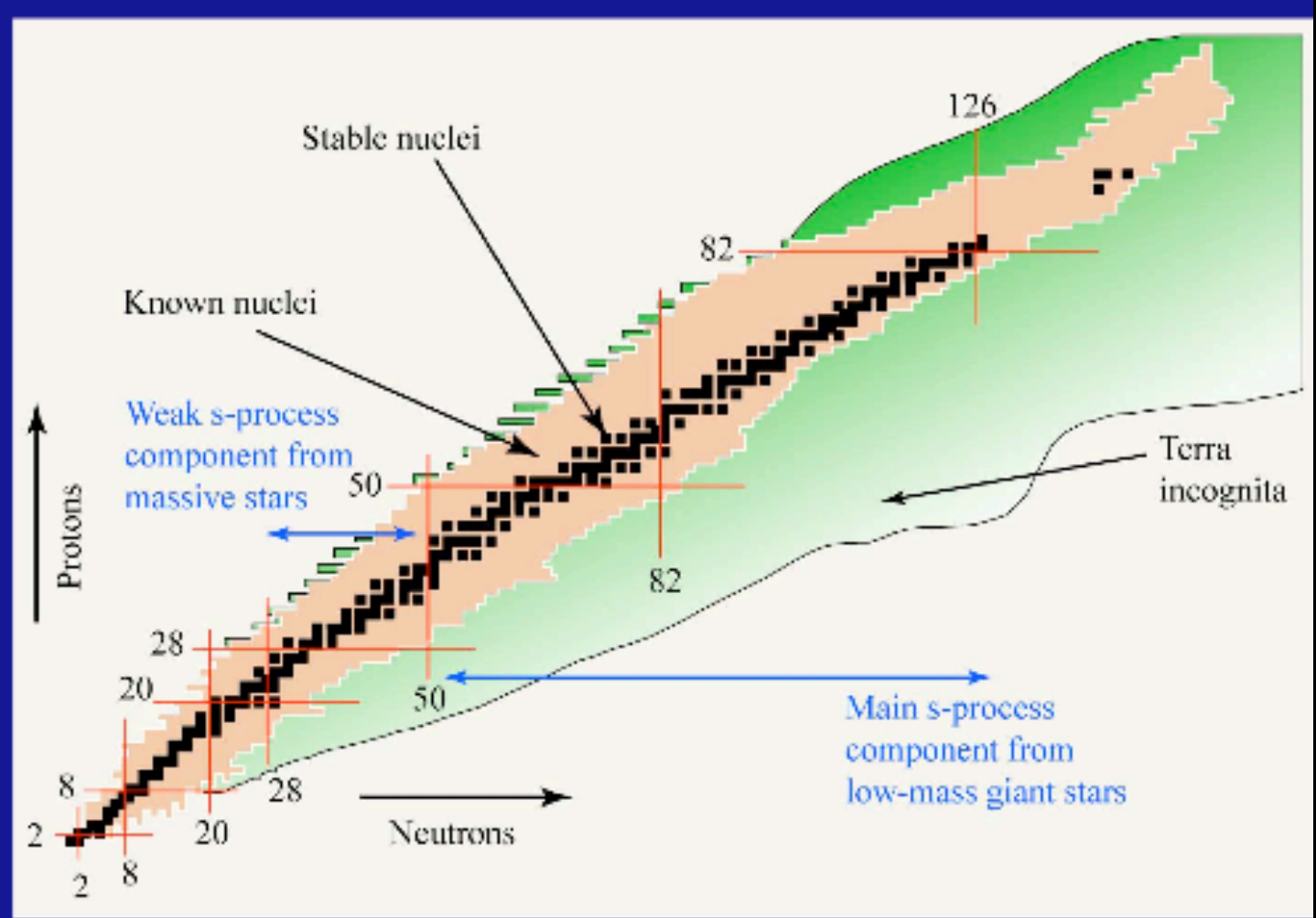

flosAlamos Falk Herwig: »Nuclear Astrophysics with Neutron Facilities« MSU - 14 Feb '05 


\section{s-process : two neutron sources}

$$
{ }^{12} \mathrm{C}+\alpha \rightarrow \mathrm{n}+{ }^{16} \mathrm{O}
$$

$$
{ }^{22} \mathrm{Ne}+\alpha \rightarrow \mathrm{n}+{ }^{25} \mathrm{Mg}
$$

- n-release during the interpulse phase

- radiative conditions, T8 $>0.9$

- low-n densities : $\mathrm{Nn}<10^{7} \mathrm{~cm}^{-3}$

- no activation of branchings

- n-release in intershell during TP

- convective conditions, T8 $>2.5$

- high-n densities : $\mathrm{Nn}<10^{10} \mathrm{~cm}^{-3}$

- activation of branchings 


\section{Radiative s-process in AGB stars}

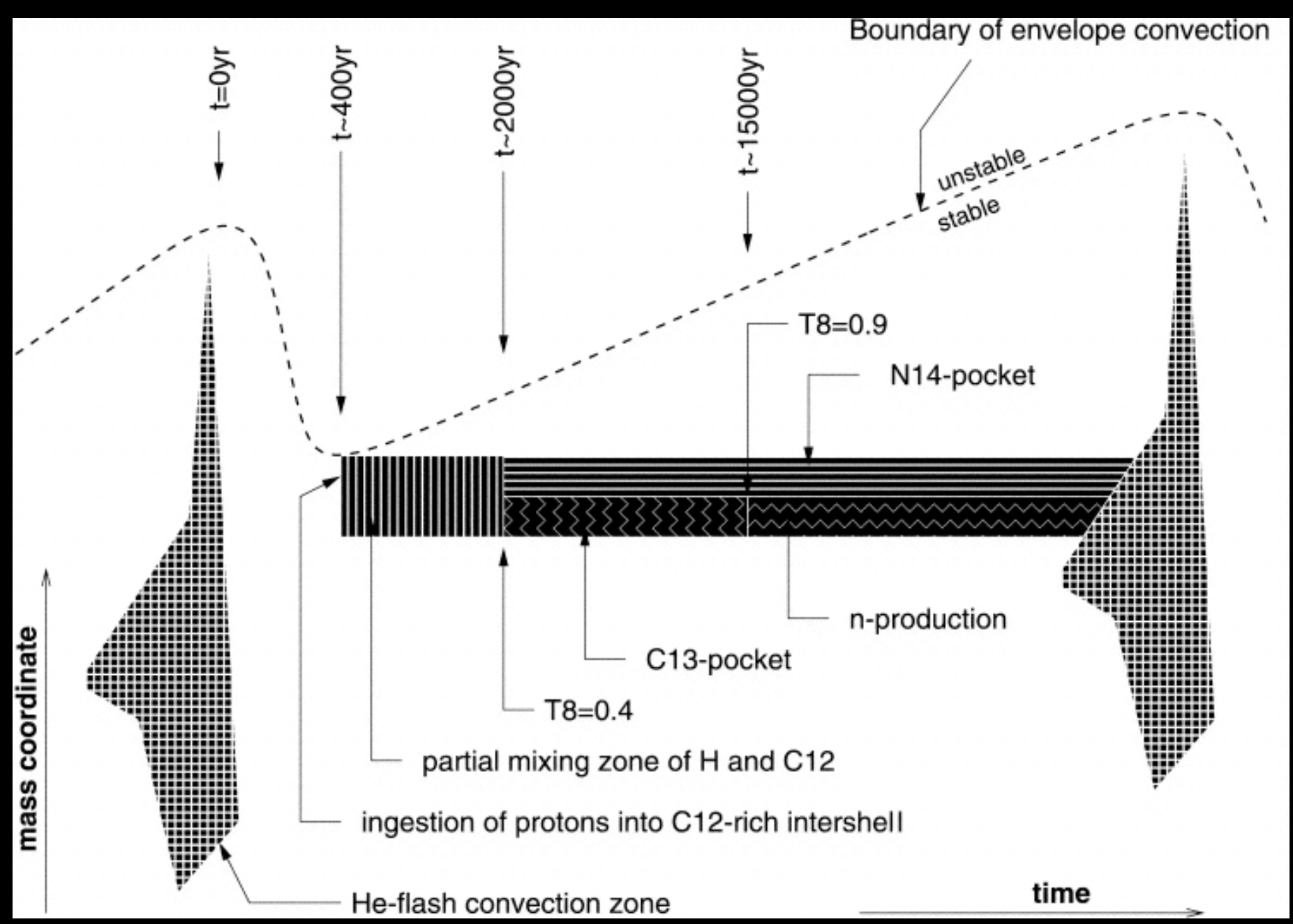




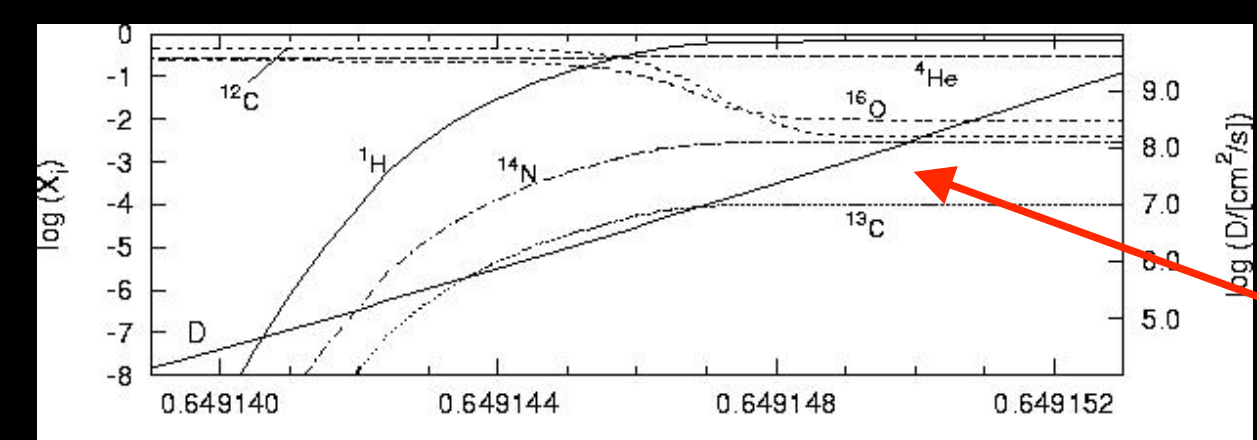

\section{Convective ov}

$500 y r$ after the He-flash

After the end of the DUP episode

D : overshoot of the envelope convection

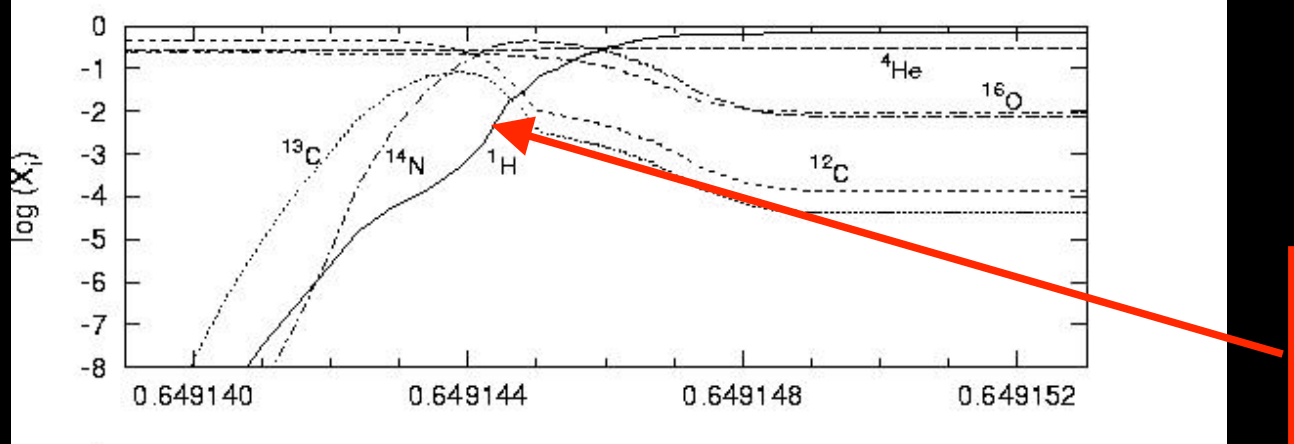

$1800 y$ r after the He-flash HBS has set in again

$\mathrm{M}\left({ }^{13}\right.$ Cpocket $) \sim 2$ to 4 x $10^{-7} \mathrm{M}_{\odot}$

S-process element distribution requires $\mathrm{M}\left({ }^{13}\right.$ Cpocket $) \sim 1$ to $2 \times 10^{-5} \mathrm{M}_{\odot}$

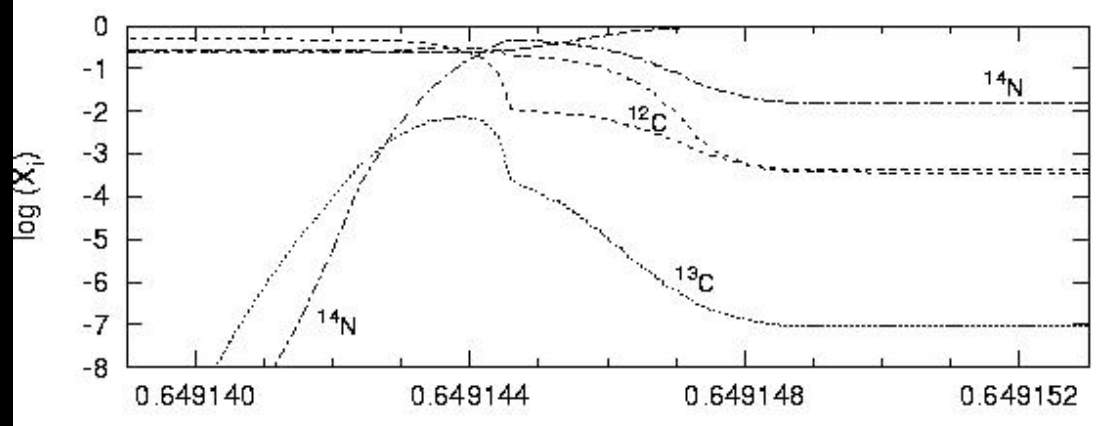

Very end of the interpulse

${ }^{13} \mathrm{C}$ has already been destroyed

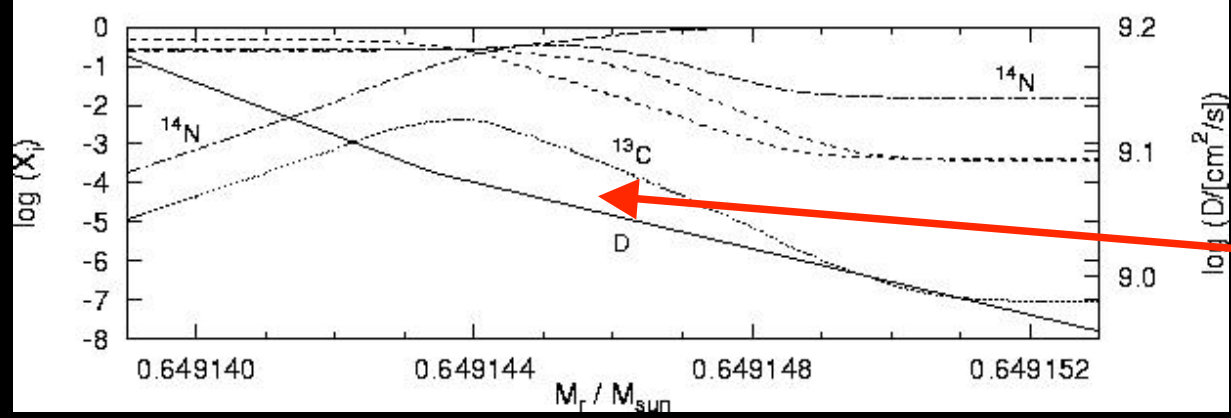

Onset of the next thermal pulse

$\mathrm{D}$ : upper overshoot zone of the TP

Herwig (2000) 


\section{$3 \mathrm{M}_{\odot}, \mathrm{Z}_{\odot}$}

Abundance profiles in the partial mixing zone at 3 times during the interpulse
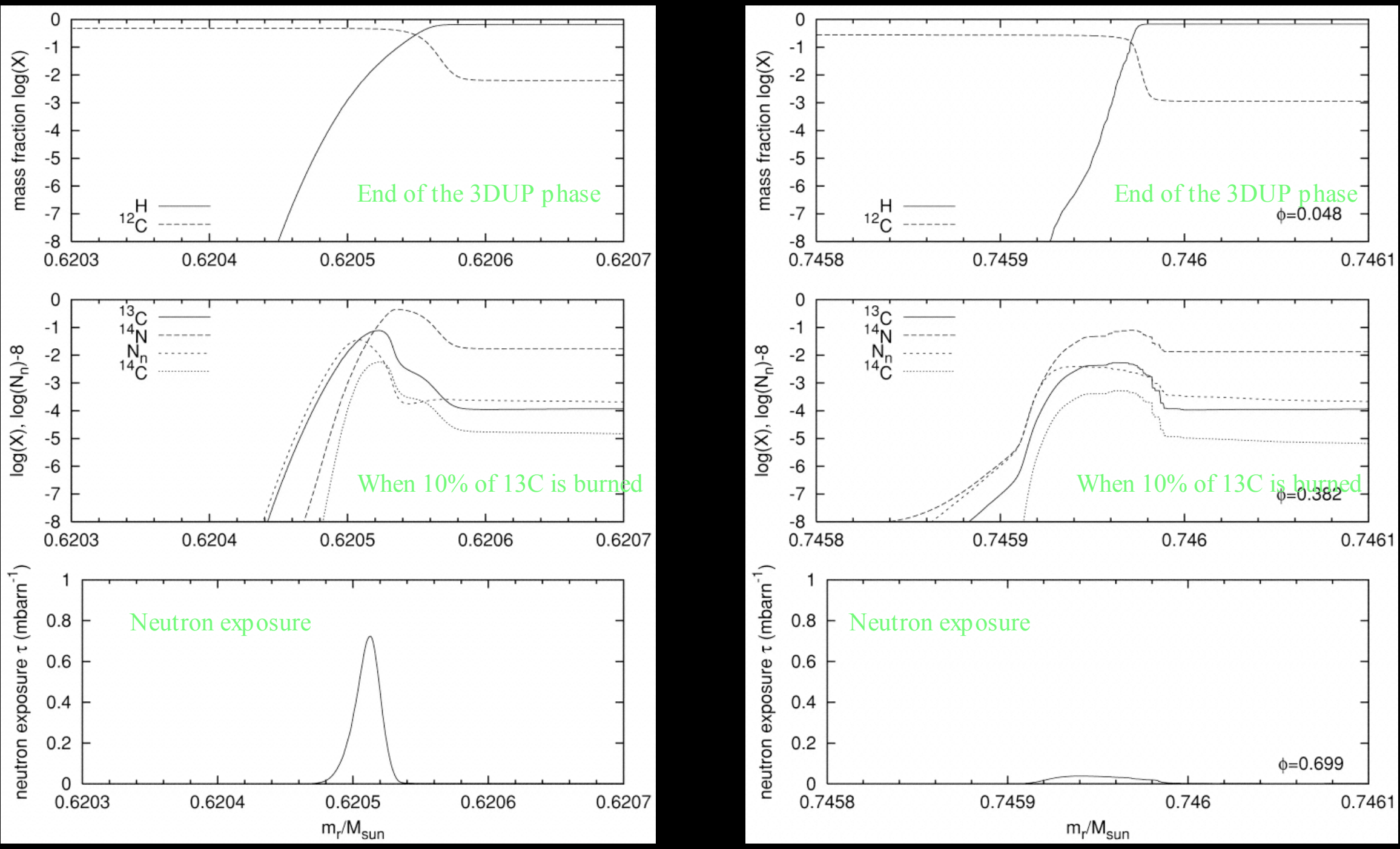

Overshoot and no rotation

Seventh TP

Rotation and no overshoot 25 th TP 


\section{Bare Core}

\section{Envelope Ejection}

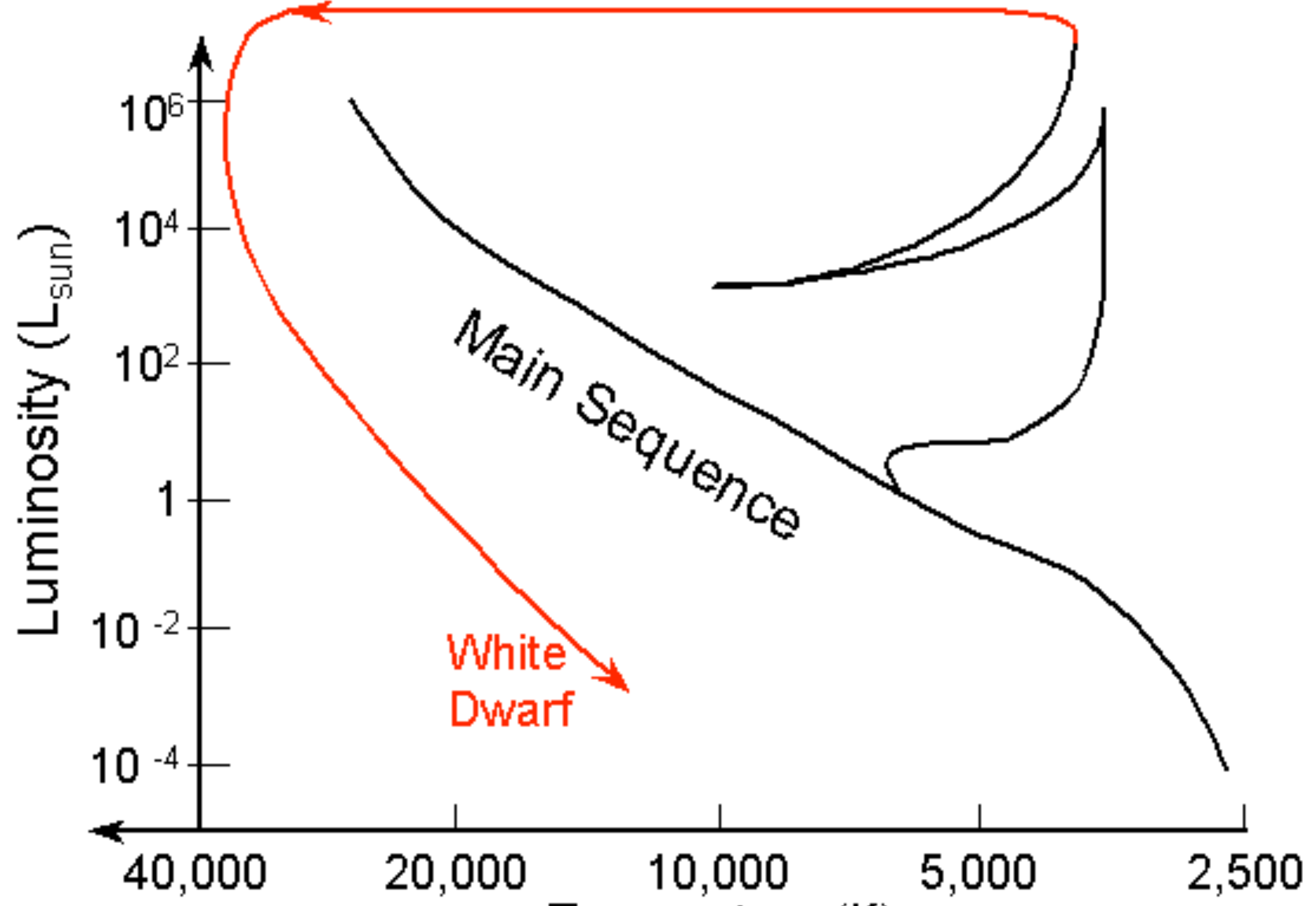

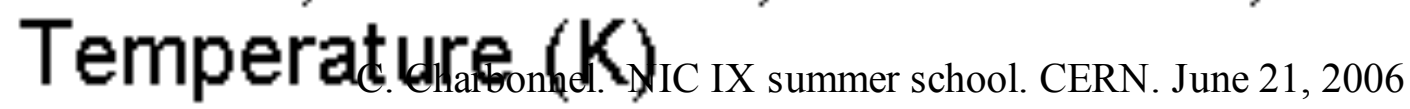




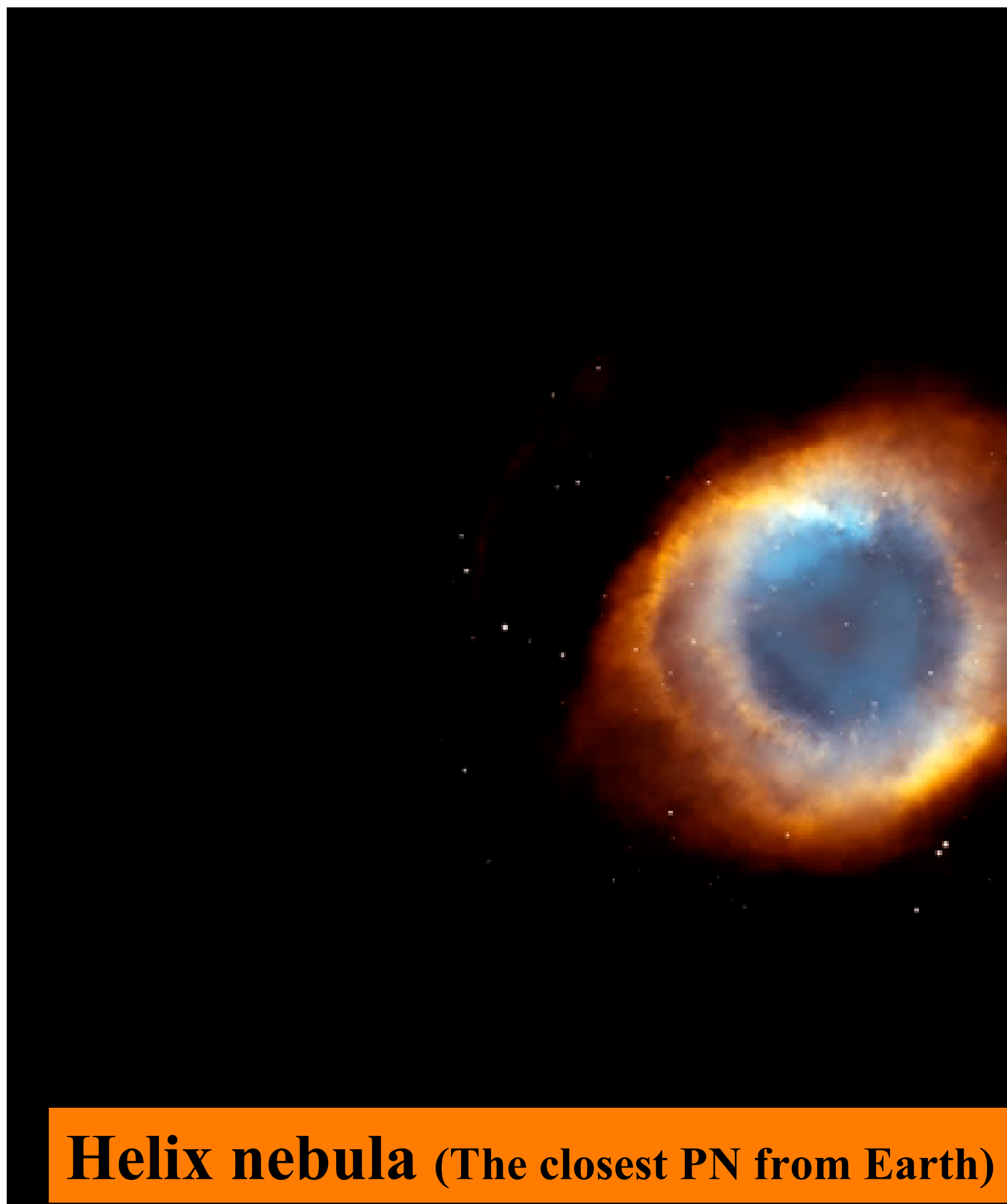




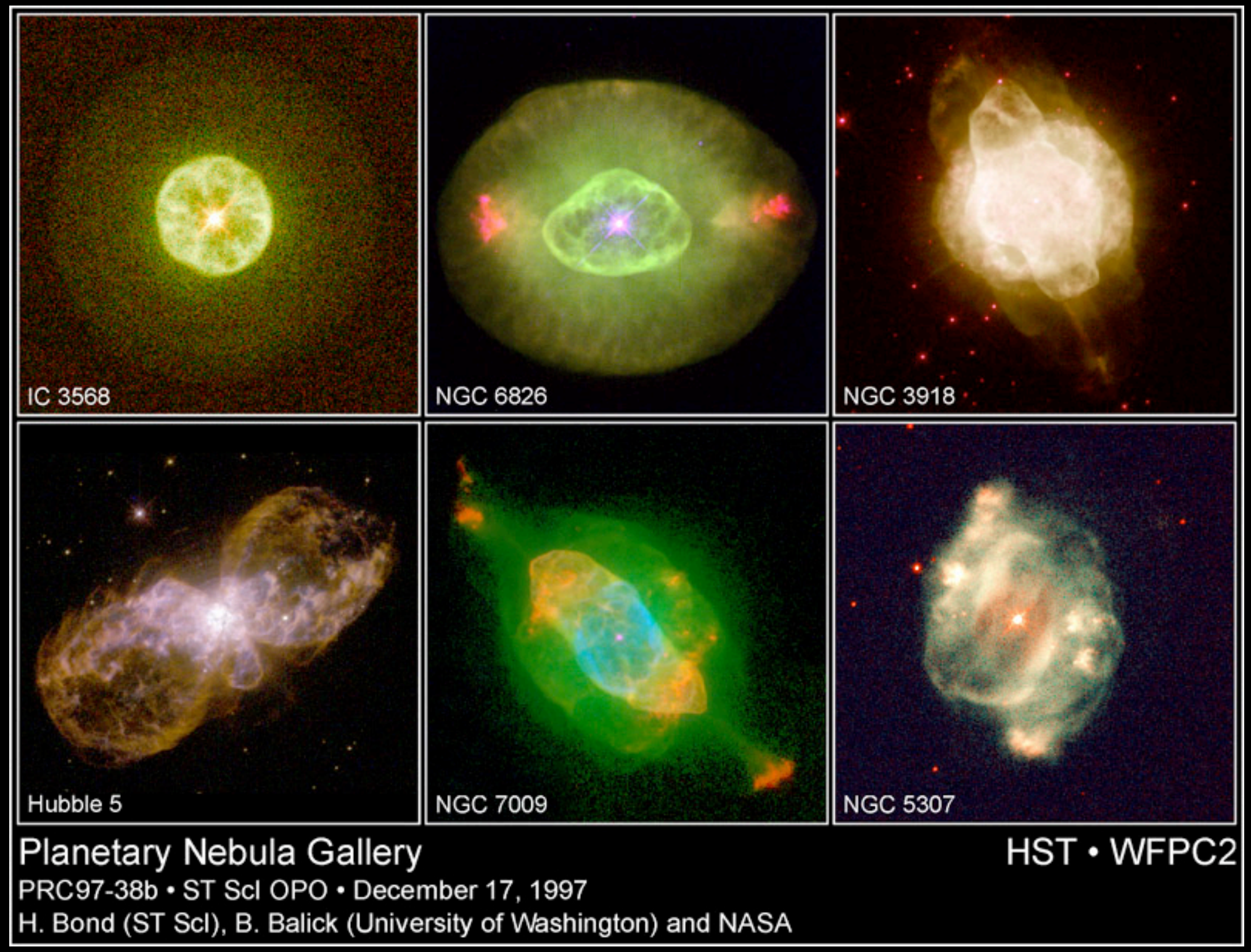




\section{White dwarf}

- Form as the outer layers of a low-mass red giant star puff out to make a planetary nebula.

- Since the lower mass stars make the white dwarfs, this type of remnant is the most common endpoint for stellar evolution.

- If the remaining mass of the core is less than 1.4 solar masses, the pressure from the degenerate electrons (called electron degeneracy pressure) is enough to prevent further collapse. 


\section{White dwarf density}

- Because the core has about the mass of the Sun compressed to something the size of the Earth, the density is tremendous: around $10^{6}$ times denser than water (one sugarcube volume's worth of white dwarf gas has a mass $>1$ car)!

- A higher mass core is compressed to a smaller radius so the densities are even higher.

- Despite the huge densities and the "stiff" electrons, the neutrons and protons have room to move around freely : they are not degenerate. 


\section{Radius of a white dwarf}

Fraknoi/Morrison/Wolff, Voyages Through the Universe, 2/e

Figure 22.1 Relating Masses and Radil of White Dwarfs

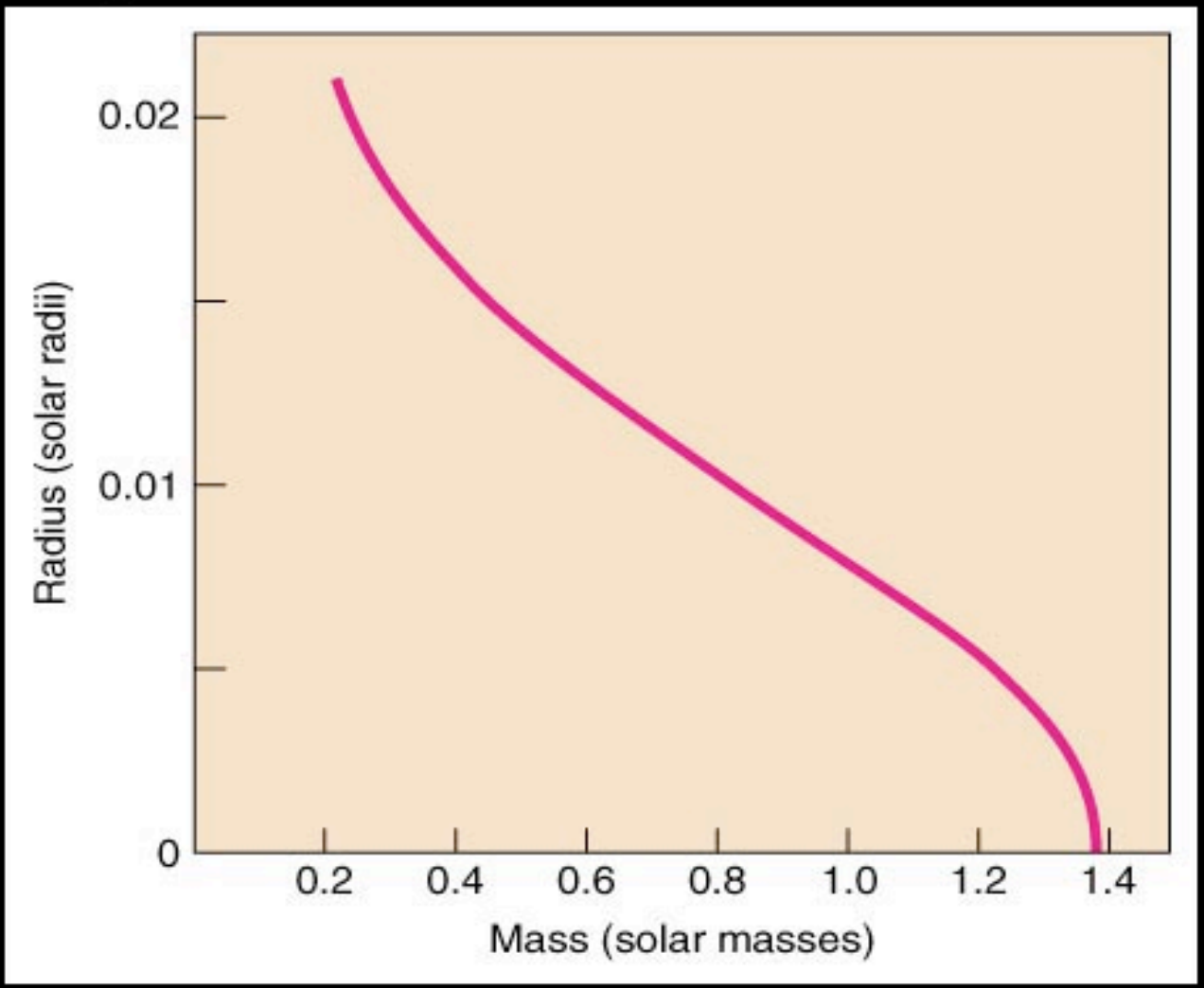

Harcourt, Inc. items and derived items copyright $\odot 2000$ by Harcourt, Inc. 


\section{White dwarf cooling}

- White dwarfs shine simply from the release of the heat left over from when the star was still producing energy from nuclear reactions

- There are no more nuclear reactions occurring so the white dwarf cools off from an initial temperature of about $100,000 \mathrm{~K}$

- The white dwarf loses heat quickly at first cooling off to $20,000 \mathrm{~K}$ in only about 100 million years, but then the cooling rate slows down:

it takes about another 800 million years to cool down to $10,000 \mathrm{~K}$ and another 4 to 5 billion years to cool down to the Sun's temperature of $5,800 \mathrm{~K}$ 


\section{White dwarf cooling}

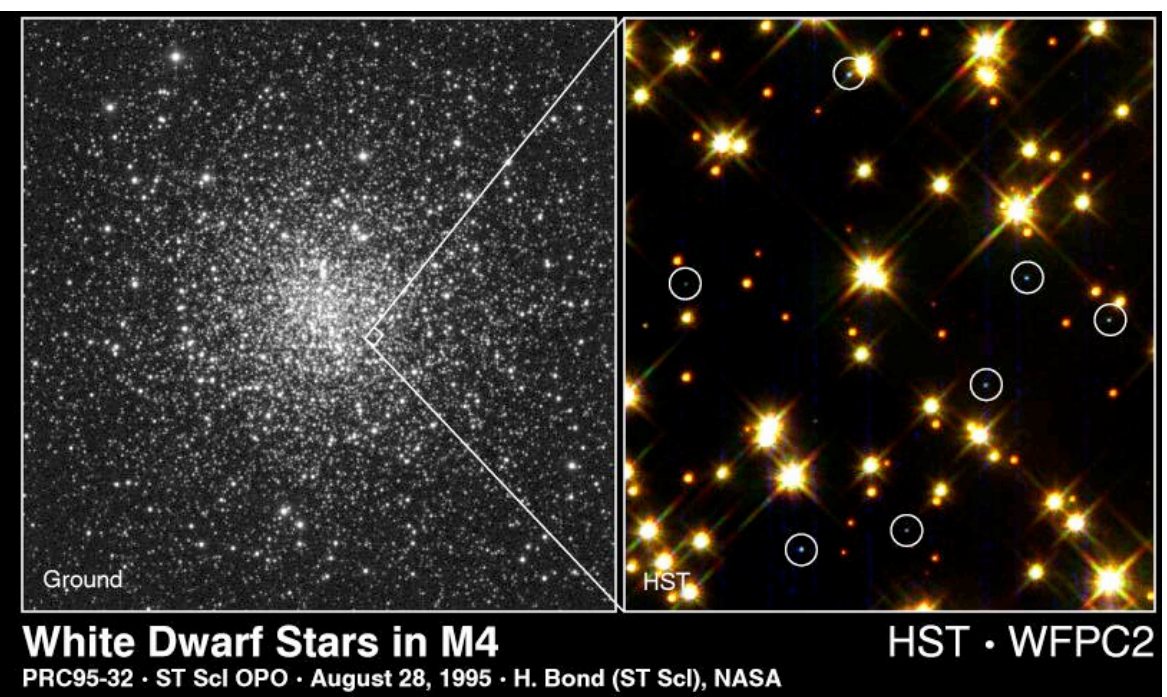

- Their rate of cooling and the distribution of their current temperatures can be used to determine the age of our galaxy or old star clusters that have white dwarfs in them

- However, their small size makes them extremely difficult to detect

- Because it is above the atmosphere, the HST can detect these small dead stars in nearby old star clusters called globular clusters

- Analysis of the white dwarfs may provide an independent way of measuring the ages of the globular clusters and provide a verification of their very old ages derived from main sequence fitting 


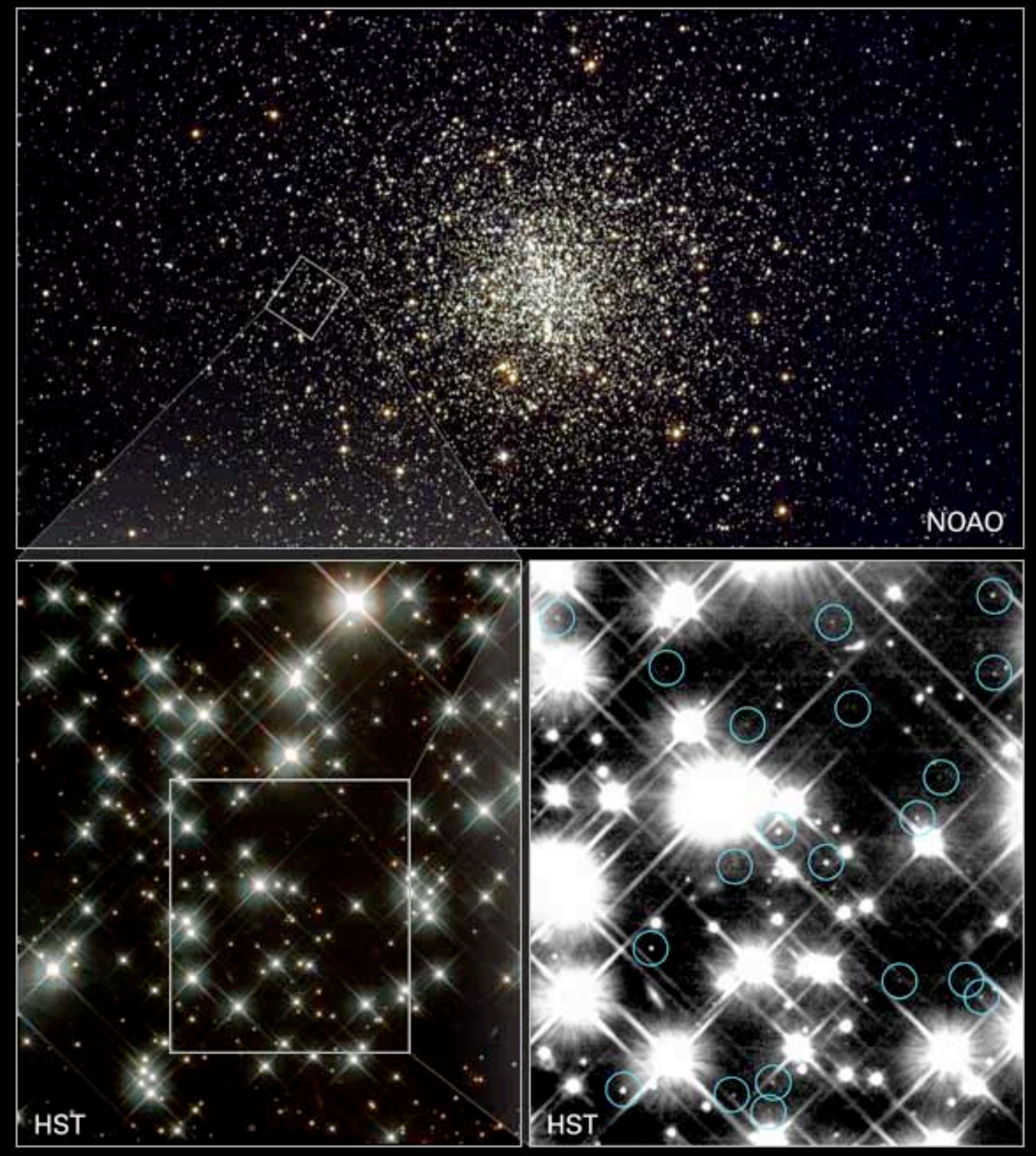

White Dwarf Stars in Globular Cluster M4

HST WFPC2

NASA and H Richer (University of British Columbia) STScl-PRC02-10

C. Charbonnel. NIC IX summer school. CERN. June 21, 2006 


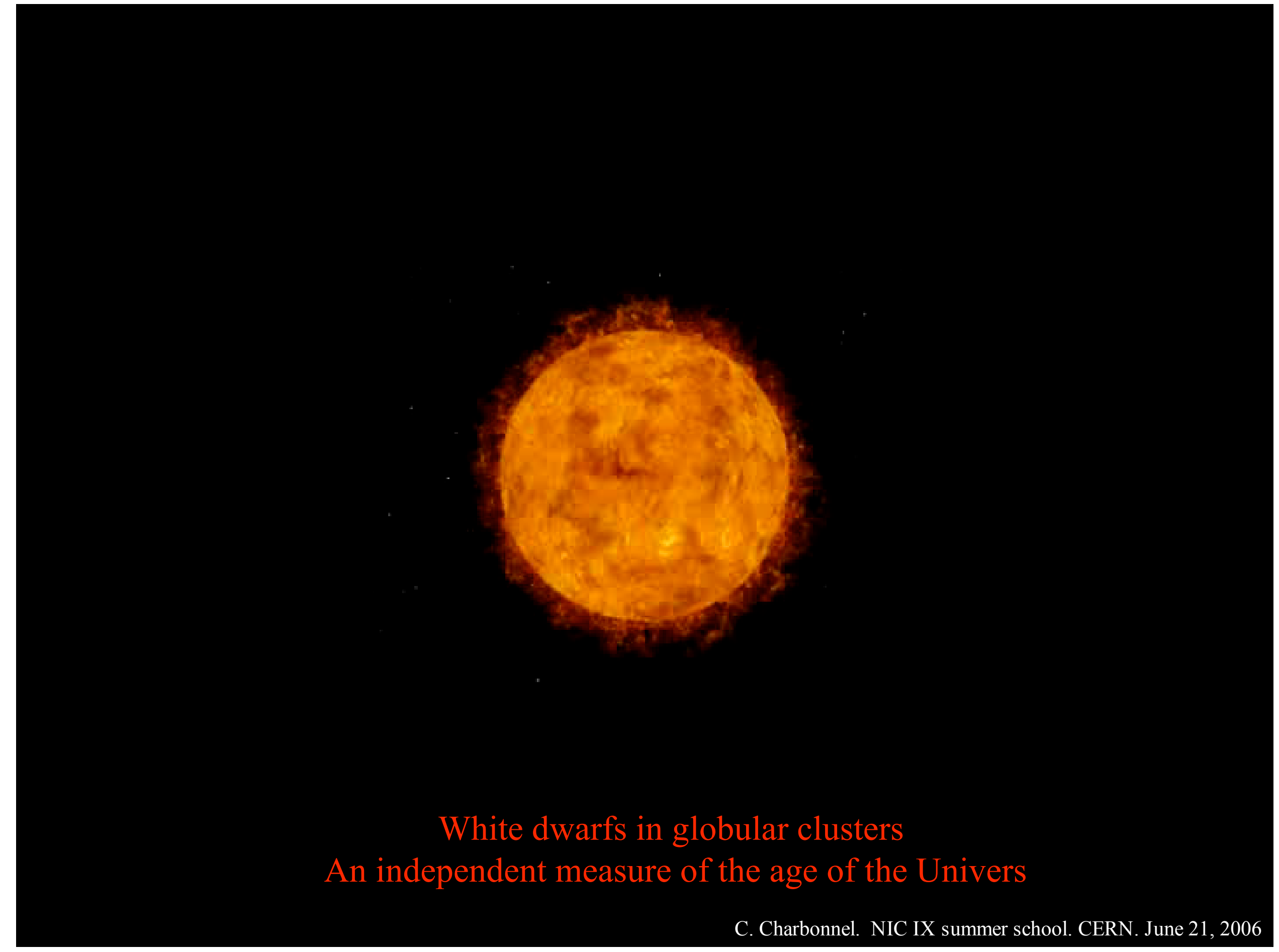

